\title{
The landscape of antigen-specific $T$ cells in human cancers
}

Bo Li1 ${ }^{1,2,7,}$, Longchao $\mathrm{Liu}^{3,7}$, Jian Zhang ${ }^{4}$, Jiahui Chen ${ }^{1}$, Jianfeng $\mathrm{Ye}^{1}$, Alexander Filatenkov ${ }^{3}$, Sachet Shukla ${ }^{5}$, Jian $\mathrm{Qiao}^{3}$, Xiaowei Zhan ${ }^{6}$, Catherine $\mathrm{Wu}^{5}$, Yang-Xin $\mathrm{Fu}^{3, *}$

1. Lyda Hill Department of Bioinformatics, UT Southwestern Medical Center, Dallas, TX, USA

2. Department of Immunology, UT Southwestern Medical Center, Dallas, TX, USA

3. Department of Pathology, UT Southwestern Medical Center, Dallas, TX, USA

4. Beijing Institute of Basic Medical Sciences, Beijing, 100850, China

5. Medical Oncology, Dana-Farber Cancer Institute, Harvard Medical School, Boston, MA, USA

6. Department of Clinical Science, UT Southwestern Medical Center, Dallas, TX, USA

7. These authors contribute equally

*: corresponding authors

Lead contact: Bo Li <bo.li@utsouthwestern.edu>

Key words: antigen-specific T cell, tumor-infiltrating T lymphocyte, single-cell sequencing, tissueresident memory signature, cancer-associated antigen, humanized mouse, TCR repertoire sequencing, non-invasive cancer diagnosis 


\begin{abstract}
Antigen-specific T cells can be orchestrated to kill cancer cells in immunotherapies but the utilities of the TCR information have not been fully explored. Here, we leveraged previous efforts on tumor TCR repertoire, and developed a novel algorithm to characterize antigen-specific TCR clusters. Joint analysis with gene expression revealed novel regulators for T cell activation. Investigation of single-cell sequencing data revealed a novel subset of tissue-resident memory $\mathrm{T}$ cell population with elevated metabolic status. Integrative analysis of TCR clusters with HLA alleles and cancer genomics data identified candidate antigens derived from missense mutations, frameshift indels, and tumor-associated gene overexpression. Predicted antigen HSFX1 was further validated using vaccinated humanized HLA-A*02:01 mice. Finally, high abundant cancer-associated TCRs were observed in the blood repertoire of early breast cancer patients, suggesting new avenues for noninvasive early detection. Thus, our analysis identified cancer-associated T cells with broad utilities in immune monitoring and cancer immunotherapies.
\end{abstract}




\section{Introduction}

Antigen-specific tumor-infiltrating T lymphocytes (TIL) play a central role in cancer immunity ${ }^{1-3}$, with demonstrated applications in cancer immunotherapies, including checkpoint blockade ${ }^{4-6}$ and adoptive cell transfer therapies ${ }^{7,8}$. Therefore, identification of antigen-specific TIL is critical to understanding tumor-immune interactions and designing individualized treatments. However, this task remains challenging despite extensive clinical efforts ${ }^{9,10}$. First, cancer antigens may come from diverse sources, including missense mutations ${ }^{11,12}$, frameshift insertions or deletions ${ }^{13,14}$, tissue specific gene overexpression ${ }^{15,16}$, and other antigenic processes ${ }^{17-20}$, making it difficult to profile all the possible targets. In addition, the antigen-binding CDR3 region on the T cell receptor (TCR) is extremely diverse ${ }^{21}$, and their targets are usually unknown. Thus limited progress has been made in the analysis of TIL repertoire despite pressing clinical needs. This is because statistical significance is usually difficult to reach in such analysis unless using a large cancer cohort and a proper computational method, neither of which is currently available to study the tumor antigen-specific T cells.

Efforts have recently been made to partition the immune repertoire into groups linking to antigenspecificity (GLIPH) ${ }^{22}$, or evaluate the similarity of CDR3s with known specificity for functional predictions (TCRdist) ${ }^{23}$. However, TCRdist prediction relies on established antigen-binding TCR repertoire, which is usually unavailable for cancer studies, while GLIPH is benchmarked for infectious disease, and we demonstrated its suboptimal specificity to accommodate the extreme diversity of tumor antigens. Therefore, due to the complicated interactions between cancer and tumor-reactive TILs, more specific strategy is required to study the repertoire of cancerassociated TCRs for improved immune monitoring and immunotherapies.

In this work, we systematically identified the antigen-specific T cells using a novel CDR3 dataset profiled from over 9,700 tumor RNA-seq samples from the Cancer Genome Atlas (TCGA) and a new computational method for highly specific grouping of TCR CDR3 sequences. These unique resources allowed us to integratively analyze the antigen-specific TILs together with cancer genetic alterations, gene expression patterns, patient clinical profiles, single-cell RNA-seq data and immune repertoire sequencing data ${ }^{24,25}$ from the public domain. This pan-cancer analysis led to several interesting findings, which might not only provide novel targets for late stage cancer treatment, but also point to new avenues for preventive cancer screens. Specifically, our analysis identified a number of metabolic enzymes as potential negative regulators for $\mathrm{T}$ cell activation and revealed a novel tissue-resident memory $\mathrm{T}$ cell subpopulation for a subset of antigen-specific TILs. We also predicted candidate cancer antigens derived from somatic alterations or overexpressed cancer-associated genes with in vivo validations, which may expand the current pool of cancer antigens for future vaccination development. Finally, we developed a predictor from the antigenspecific CDR3s to distinguish cancer patients from healthy individuals, and demonstrated its potential application to non-invasive early cancer detection or immune monitoring. 


\section{Results}

\section{Detection of antigen-specific CDR3 clusters with iSMART}

We have previously described the TRUST algorithm ${ }^{26}$ for sensitive detection of $\mathrm{T}$ cell receptor hypervariable CDR3 sequences using bulk tissue RNA-seq data. In this work, we applied a later version of TRUST ${ }^{27}$ with improved sensitivity to 9,709 TCGA tumor RNA-seq samples and assembled 1.5 million CDR3 sequences (Figure 1a). Of these, 170,000 were complete productive CDR3s, following the IMGT nomenclature ${ }^{28}$. A sizeable fraction of the human $\mathrm{T}$ cell repertoire is public, derived from biased V(D)J recombination ${ }^{29}$, and are present in both healthy and diseased individuals. To exclude the irrelevant public TCRs, we compared the TCGA TIL CDR3s with a large cohort of TCR repertoire data from non-cancer individuals ${ }^{30}$ (Methods). CDR3s observed in these samples with high abundances were excluded, leaving 82,000 non-public sequences.

Identification of antigen-specific CDR3 groups from TCR repertoire data is highly desirable, yet challenging due to the high diversity of CDR3 regions ${ }^{21}$ and promiscuous binding between $\mathrm{T}$ cell receptors and antigenic peptides ${ }^{31-33}$. A previous work, GLIPH ${ }^{22}$, demonstrated that CDR3s grouped into motif-sharing clusters are expected to recognize the same antigens. In our benchmark analysis we observed GLIPH groups a substantial fraction of CDR3s of different antigens (Supplementary Figure 1), thus might not be optimal for analyzing TIL TCR data. Therefore, we developed a new method, immuno-Similarity Measurement by $\underline{\text { Aligning Receptors }}$ of T cells, or iSMART, with increased clustering specificity (Methods). In brief, iSMART performs a specially parameterized local alignment on CDR3s, builds a pairwise comparison matrix and divides it into clusters with highly similar sequences. We benchmarked iSMART without variable (V) gene assignment, because 1) a large fraction of the TRUST assemblies do not have V gene information and 2) GLIPH does not rely on V gene assignment for clustering. We tested both methods using a curated CDR3 database containing the experimentally validated, antigen-specific TCR sequences ${ }^{34}$ (Methods).

We first applied iSMART to the 2,347 curated CDR3s specific to 15 selected antigens (Supplementary Table 1), and observed more specific grouping compared to GLIPH measured by cross-classification errors (Figure 1b). Overall GLIPH clustered a higher percentage of total CDR3s (29\%) than iSMART (17\%), but iSMART achieved significantly higher specificity $(\mathrm{p}=0.00078$, Wilcoxon rank sum test) measured by cluster purity (Figure 1c) and called more clusters with unique antigen assignments (Figure 1d). As our goal is to identify tumor-specific CDR3s, the higher specificity of iSMART is a desirable feature. Therefore, we applied it to the 82,000 non-public CDR3 sequences and detected 4,501 clusters (Figure 1a). As most clusters contain more than one individuals, we also used the term 'CDR3 cluster' to denote the subset of patients carrying the CDR3s in a given cluster. A total of 15,254 CDR3 sequences were grouped into these clusters, and were referred as cancer-associated CDR3s.

\section{Features of CDR3 clusters and association with tumor gene expression profiles}

The number of sequences in the clusters spans two orders of magnitude (Supplementary Figure 2a), and for each sample, the number of clustered CDR3s (K) also spans two orders of magnitude (Supplementary Figure 2b). For each gene, we calculated the partial Spearman's correlation between $\boldsymbol{K}$ and its expression levels (Supplementary Table 2), controlled for tumor purity, which is expected to influence both values ${ }^{35}$ (Methods). Among the genes with top positive correlations are putative T cell activation markers, including TBX21 (T-bet), ICOS, TIGIT and granzymes (Supplementary Figure 3a). Gene ontology enrichment ${ }^{36}$ analysis suggested that the top 500 genes are strongly enriched for immune cell activation and immune responses (Supplementary 
Figure 3b). Interestingly, on the top of the list there is a pair of lysophosphatidylserine receptors, GPR174 and P2RY10, which have been identified as suppressors for regulatory T cell function ${ }^{37}$ (Supplementary Figure 3a). These results strongly suggest that CDR3s clustered by iSMART are enriched for activated T cells in the tumor microenvironment.

We next investigated genes negatively correlated with $\boldsymbol{K}$ as potential regulators for $\mathrm{T}$ cell inactivation and exclusion (Supplementary Figure 4). Of the 414 genes with correlation $<-0.1$ and FDR $<0.05$ in at least 3 cancer types, we observed 4 interesting clusters. Cluster i) contains a putative oncogene $M A P K 3^{38}$, inhibition of which has been linked to enhanced anti-tumor immune response $^{39}$. This cluster also harbors a key glycolysis enzyme, $A L D O A$, which has recently been shown to impair T cell infiltration and cytotoxicity ${ }^{40}$. Cluster ii) contains two oncogenes, $R H O D$ and $P K P 3$, the former recently being implicated in immune suppression ${ }^{41}$. We also identified a number of other metabolic enzymes, including protein metabolic enzyme POMGNT1, cytochrome c enzymes COX6A1 and $U Q C R Q$, lipid metabolic enzymes DGAT1 and FAAH, etc, supporting the recently elucidated immunosuppressive role of cancer metabolism pathways ${ }^{42}$.

\section{Identification of tissue-resident memory $\mathrm{T}$ subpopulations with distinct metabolic status}

To further elucidate the phenotypes of the T cell clonotypes with clustered CDR3s, we analyzed a recently generated single cell RNA-seq (scRNA-seq) data with matched TCR information ${ }^{43}$. Using the TCGA-derived CDR3s as clonotype markers, we identified a number of clustered $\mathrm{T}$ cell clones in the 3 breast tumor scRNA-seq samples. We first studied sample BC10, which has the largest amount $(n=55)$ of cells carrying clustered CDR3s. The selected 55 cells were visualized on the background of all 4,926 cells using t-Distributed Stochastic Neighbor Embedding (tSNE) ${ }^{44}$ plots, and observed a local clustering of 18 events in a restricted region (Figure 2a). All 18 events share the same $\beta$ chain CDR3 sequence, and we delineated the region containing these cells as a separate CD8+ subgroup ( $n=44)$. Differential gene expression analysis on the cells in this group against all the others (Supplementary Table 3 ) revealed up-regulated genes both involved in T cell cytotoxicity (GZMB, PRF1, IFNG) and exhaustion (PDCD1, LAG3) (Supplementary Figure 5). Interestingly, the top targets showed high consistency with a recently reported tissue resident memory $\mathrm{T}$ cells $\left(\mathrm{T}_{\mathrm{rm}}\right.$ ) signature ${ }^{45}$, including up-regulation of CD103 (ITGAE), TIGIT, GZMB, and down-regulation of SELL (CD62L), KLF2 and KLRG1. This group also expresses a number of other previously reported Trm markers ${ }^{46}$ (Supplementary Figure 6), including transcription factor ZNF683, or HOBIT (homolog of Blimp-1 in T cells), a key regulator for $\mathrm{T}_{\mathrm{rm}}$ differentiation ${ }^{47}$. We observed significant association of ZNF683 expression with better outcomes in multiple cancer types (Figure $\mathbf{2 b}$ ), supporting the anti-tumor role for $\mathrm{T}_{\mathrm{rm}}$ cells.

T cells undergo profound differentiations in the tumor microenvironment, and it is unclear which evolutionary path $\mathrm{T}$ cells have taken to become resident memory cells. The 44 cells in the subgroup come from 20 productive clonotypes, which in total contain 418 cells. We performed single cell trajectory analysis ${ }^{48}$ to infer the progression of these TILs (Figure 2c and Methods). The pseudotime trajectory starts from a group of precursor cells ( $\mathrm{T}_{\text {pre }}$ ) expressing high levels of $I L 7 R, S E L L$, and KLRG1, with low expression of effector molecules (GZMB, PRF1, IFNG) and exhaustion markers (PDCD1, LAG3, TIM-3). These markers agree with the signatures of T cells newly entering the tumor microenvironment, thus confirming the pseudo temporal ordering. Two clusters were observed at the end of the trajectory, both carrying the resident memory markers, and we named them $\mathrm{T}_{\mathrm{rm} 1}$ and $\mathrm{T}_{\mathrm{rm} 2}$. Notably, the $\mathrm{T}_{\mathrm{rm} 1}$ cluster largely overlaps with the previously identified CD8+ subgroup. Differential expression analysis revealed that comparing to $\mathrm{T}_{\mathrm{rm} 1}$, the newly identified $\mathrm{T}_{\mathrm{rm} 2}$ population upregulates genes significantly enriched in the oxidative 
phosphorylation (OXPHOS) process (FDR=6.77×10-33), featured by GAPDH, COX8A and MT-CO2 (Figure 2d). Pseudotime trajectories for individual clonotypes revealed that the differentiation of $\mathrm{T}$ cells into resident memory status is receptor dependent (Supplementary Figure 7). Specifically, we observed two modes of evolution: majority of clonotypes evolve from $T_{\text {pre }}$ into $T_{\mathrm{rm} 1}$, with others from $\mathrm{T}_{\mathrm{rm} 1}$ to $\mathrm{T}_{\mathrm{rm} 2}$. Direct differentiation of $\mathrm{T}_{\text {pre }}$ into $\mathrm{T}_{\mathrm{rm} 2}$ was not observed.

We analyzed other scRNA-seq samples to see if this observation is reproducible, and indeed, a strikingly similar pseudotime trajectory for resident memory T cells was observed in sample BC11 (Supplementary Figure 8a). Representative markers observed in sample BC10 also showed significant differences across the 3 cell groups with consistent trends. Higher expression of OXPHOS genes was also observed in $\mathrm{T}_{\mathrm{rm} 2}$ group (Supplementary Figure 8b). In addition, the corresponding clonotypes also displayed two evolutionary patterns (Supplementary Figure 8c), consistent with our findings for $\mathrm{BC} 10$. These results indicate that the $\mathrm{T}_{\mathrm{rm}}$ cells further divide into two populations distinguished by low or high metabolic status, and the differentiation from their precursors into these populations is dependent on the $\mathrm{T}$ cell receptors.

\section{Identification of novel cancer neoantigen candidates}

Computational identification of cancer neoantigens relies on the prediction of peptide binding to HLA alleles ${ }^{49,50}$, while little is known whether the predicted binders can elicit an immune response. Having studied the phenotypes of the clustered T cell clonotypes using single-cell sequencing data, we next sought to approach this problem from the $\mathrm{T}$ cell receptor angle using the iSMART-derived CDR3 clusters and TCGA cancer genomics data. We have previously provided a proof-of-concept analysis to statistically identify novel candidate neoantigens ${ }^{26}$. In this work, we extended this effort by searching for co-occurrence of somatic mutations and CDR3 clusters (Methods). First, each of the 6,136 recurrent $(n \geq 3)$ missense mutations was paired with each of the CDR3 clusters, with statistical significance evaluated using random permutations. At FDR $<0.05$, we identified 6 significant pairs with at least 2 individuals carrying both the mutation and the CDR3 sequence (Figure 3a). 4 of them (excluding the two CD163L mutations) are predicted to generate HLA binding peptides. Individuals carrying two of these mutations, GLIS3 S47L and SLITRK3 E968K, have matched HLA genotypes (Figure $\mathbf{3 b}$ and Supplementary Table 4).

It has been implicated that tumor frameshift insertions or deletions (indels) may generate neopeptides to trigger immune responses ${ }^{14}$. We applied the same analysis to study the 1,225 recurrent $(n \geq 3)$ frameshift indels. Compared with missense mutations, we observed more significant pairs $(n=10)$ with fewer indels, indicating that frameshift indels might be another important source of neoantigens. As aberrant mRNA products are subject to nonsense-mediated decay ${ }^{51}$, we only focused on these $(n=4)$ with frameshift alleles confirmed in the RNA-seq data (Figure 3c). The top target is a one-base deletion in gene AXIN2, generating a 23 amino acids neopeptide (Figure 3d). We predicted HLA binding for the neopeptide, and identified two closely related 9-mers to bind to the HLA alleles of the mutation carriers (Figure 3e). Besides AXIN2, two other indels on DYRK4 and RNF43 also generate neopeptides predicted to bind to HLA alleles matching the genotypes of the carriers (Supplementary Figure 9). We observed that all the related individuals were stomach cancer patients with high levels of microsatellite instability $(\mathrm{MSI})^{52}$, and the indels all occurred in the short tandem repeat regions. It is known that patients with DNA mismatch repair (MMR) deficiency, a cause for MSI, have better responses to checkpoint blockade therapies ${ }^{53}$. Our results corroborate this clinical observation by identifying a number of potentially immunogenic neoantigens resulted from MMR deficiency, and demonstrated that iSMART can prioritize TCRs associated with neoantigens derived from genetic alterations. 


\section{Identification of novel cancer associated antigen candidates}

Most current studies focus on searching for tumor antigens from mutated genes with matched HLA alleleotypes combining the elution of peptides from the MHC molecules ${ }^{54}$. However, malignant cells may overexpress a number of genes that are usually silenced in most normal tissues, resulting in novel antigenic targets for cancer treatment. This is exemplified by the clinical use of cancer/testis antigens, which have restrictive expression in the male germ cells ${ }^{15,16}$. We performed a genome-wide differential gene expression analysis on each of the 120 qualifying CDR3 clusters, and identified a total of 1,409 significant (FDR $<0.05$ ) genes from 115 clusters (Methods and Supplementary Table 5). Of these, two clusters (1724 and 1767) showed an interesting enrichment in colon and endometrial cancers, with distinct CDR3 conservation patterns (Figure 4a). We performed differential expression analysis on the combined samples from the two clusters, and identified Heat Shock Transcription Factor X-linked 1 (HSFX1) as the top hit (Figure 4b). This gene has extremely low expression (median TPM $\leq 0.02$ ) in all the tissue types covered in the GTEx data 55 , while expressed (TPM $\geq 1$ ) in $13 \%$ colorectal and $75 \%$ endometrial cancers (Supplementary Figure 10a-b). There is over 100-fold change in the expression levels between some tumor samples and the normal tissues. It is also a favorable predictor of survival for endometrial cancer (Figure 4c). Therefore, we hypothesized that the tissue-specific overexpression for HSFX1 may be a signature for cancer-associated antigen and a trigger for anti-tumor immune response.

Of the seventeen colon or endometrial cancer samples from cluster 1724 and 1767, nine express HSFX1 and have solved HLA genotypes ${ }^{56}$ (Supplementary Figure 11a). Computational prediction for HLA allele binding suggests that HSFX1 protein generates a 9-mer peptide VMFPHLPAL as a strong binder to 3 common alleles, including HLA-A*02:01 (Supplementary Figure 11b). Interestingly, all the nine individuals carry at least one predicted HLA allele (Supplementary Figure 11c), and the probability of this observation is estimated $\leq 0.00038$ (Methods). These results strongly suggested that $H S F X 1$ might be an immunogenic cancer antigen. To validate this prediction, we synthesized the 9-mer antigen peptide (VMF) and injected it into HLA-A*02:01 humanized transgenic mice (Methods). We used peptide VRFPHLPAL, which has one amino acid difference, as control, because it is predicted not to bind HLA-A*02:01. After 18 days, splenocytes of the vaccinated mice were collected to perform an IFN $\gamma$ ELISPOT assay for antigen-specific T cell responses (Figure 5a). Compared to the control peptide (VRF), we observed significantly higher IFN $\gamma$ response in the transgenic mice but not in identically primed immunocompetent mice with $\mathrm{H}-2 \mathrm{~K}^{\mathrm{b}}$ genotype (Figure 5b-c, Supplementary Figure 12). Based on these results, we concluded that the 9-mer peptide VMF derived from HSFX1 binds to human HLA-A*02:01 allele and can elicit a $\mathrm{T}$ cell response in vivo.

In addition to HSFX1, we also identified a putative cancer/testis antigen, TSSK2, with expression restricted to esophageal and stomach tissues (Supplementary Figure 13a). TSSK2 also generates a peptide binding to multiple HLA alleles (Supplementary Figure 13b), matching the genotypes of the individuals expressing TSSK2 from cluster 189 (Supplementary Figure 13c), with probability $<0.0010$ (Methods). These results suggest that genes with tumor-specific overexpression might produce cancer-associated antigens and elicit $\mathrm{T}$ cell responses. Our analysis revealed a number of such unmutated genes as promising targets for cancer vaccine development.

\section{Potential non-invasive cancer diagnosis using cancer-associated CDR3s}


In the above analysis, we observed multiple sources of potential tumor antigens showing significant associations to the iSMART identified CDR3 clusters, suggesting that the clustered CDR3s are enriched for cancer-associated T cells. We therefore investigated whether it is possible to detect these CDR3s in the TCR repertoire from the peripheral blood mononuclear cell (PBMC) of the cancer patients. We studied a cohort of 21 late-stage melanoma patients before and after anti-CTLA4 treatment ${ }^{25}$. When compared to the healthy donors, we identified significantly higher abundance of cancer-associated CDR3s in the patients' blood samples (Figure 6a) (Methods). Using cancer-associated CDR3 counts as a disease predictor, pre- and post- PBMC samples reached similar area under curve (AUC) of 0.80 and 0.82 respectively (Figure 6b).

We next evaluated the performance of the above approach on the challenging yet more useful task of predicting early cancer status via PBMC repertoire. We applied the same method to study a cohort of 16 early-stage breast cancer samples with both PBMC and TIL repertoires sequenced ${ }^{24}$. Indeed, both repertoires showed significantly higher levels of cancer-associated CDR3s than healthy donors (Figure 6c-d), indicating that the abundance of cancer-associated CDR3s is able to distinguish healthy individuals from both late and early stage cancer. Using iSMART-clustered CDR3 counts as a predictor, we observed an AUC of 0.73 for PBMC samples (Figure 6e). With more future studies on pre-cancer TCR repertoire sequencing, this approach holds the potential to be developed into a non-invasive cancer diagnostic criterion.

The distributions of the clonal frequencies of the cancer-associated CDR3s in the PBMC samples also showed interesting differences between late and early stage tumors (Supplementary Figure 14). Specifically, melanoma PBMC samples have more cancer-associated CDR3s with medium high abundance, where early breast cancer PBMC samples have a few CDR3s with high abundance. This might be related to the known fact that in an adaptive immune response, many effector $\mathrm{T}$ cells differentiate into memory cells for long-term protection, resulting in reduced clonotype frequencies. Based on a previous study on cancer and inflammation ${ }^{57}$, we speculated that during early cancer development, the immune system is able to recognize and respond to a few shared antigens (such as HSFX1), and produce a significant amount of effector T cells in the circulation. The CDR3 sequences of these T cells may serve as diagnostic markers for preventive early cancer detection or immune monitoring. 


\section{Discussion}

Despite extensive efforts and critical clinical applications, antigen-specific TILs remain largely uncharacterized, mainly because it is experimentally challenging to identify the immunogenic cancer antigens and to profile the tumor-reactive T cells. In this work, we extracted CDR3s from the tumor RNA-seq data, and identified a large number of CDR3 clusters with high sequence similarity. Due to the excessive diversity of the TCR repertoire, the probability that different individuals independently produce near-identical non-public TCRs is extremely low, suggesting that shared antigen-specificity is the main cause for the generation of these CDR3 clusters. Previous studies have also shown that TCRs sharing motifs on the CDR3 region may recognize the same antigen ${ }^{22,23}$. Therefore, we used iSMART identified CDR3 clusters as surrogates for TCR antigen-specificity, and comprehensively analyzed the tumor-specific TILs using a large human cancer cohort.

We leveraged the iSMART-clustered clonotypes to perform an in-depth analysis of a tumor single cell RNA-seq dataset with solved T cell receptor sequences, and observed an interesting group of CD8+ T cells. The marker set for this group is highly consistent with a recent study on $\mathrm{T}_{\mathrm{rm}}{ }^{45}$, suggesting reproducible identification of $\mathrm{T}_{\mathrm{rm}}$ in triple-negative breast tumor microenvironment. Using CDR3 as clonotype markers, we further identified two subpopulations of $\mathrm{T}_{\mathrm{rm}}$ with distinct metabolic states, and observed divergent evolutionary paths to these states among different TIL clonotypes. Our results suggest that after initial homing to the target tissue, $\mathrm{T}_{\mathrm{rm}}$ may switch to a high metabolic status, featured by elevated expression of OXPHOS genes. This result is potentially linked to the immunosuppressive roles for metabolic enzymes in the malignant cells, which they use to compete resources for $\mathrm{T}$ cell survival and cytotoxic functions.

It has been shown from protein structure studies that one antigenic peptide may bind to dissimilar CDR3 sequences with different docking strategies ${ }^{58,59}$, suggesting that individuals responding to the same antigen may carry divergent TCR sequences. Indeed, we observed two distinct CDR3 sequences from clusters 1724 and 1767, which were both predicted to recognize the same antigen derived from cancer-associated antigen HSFX1. We performed in vivo experiments using transgenic humanized mice to show that a 9-mer peptide derived from a predicted antigen HSFX1 is able to bind HLA-A*02:01, and induce reliable T cell responses. These results, combined with the observation that HSFX1 has restricted expression in selected cancers, and its positive clinical relevance, strongly indicated that it might escape central tolerance in humans and become an immunogenic cancer-associated antigen. We will rely on future clinical studies using colorectal or endometrial cancer patients expressing HSFX1 to explore its potential clinical utilities.

A fraction of the CDR3 clusters remain unassociated with any potential targets, likely due to the unexplored categories of cancer-associated antigens. In our gene expression analysis, we observed significant associations of some clusters with non-coding RNAs (Supplementary Table 5), such as IncRNA, pseudogenes and small nucleolar RNAs (snoRNA). Ribosome profiling data suggests that many non-coding RNAs are actually translated ${ }^{60}$, which may serve as valid cancer antigens when overexpressed in the tumor tissues. snoRNAs participate in many biological processes, including RNA splicing. Thus, their abnormal expression in selected cancer types may produce new antigenic targets from alternative splicing. Post-translational modification (PTM) may also generate foreign peptide products that are subject to immunosurveillance ${ }^{17}$. However, due to the insufficiency of related data, it is currently challenging to study the antigenic potentials of these mechanisms in cancer immunity. 
The iSMART identified CDR3 clusters might have promising applications in cancer diagnosis. In our proof-of-principle analysis on a small patient cohort, we observed promising predictive power for early-stage breast cancers using blood TCR repertoire data. This result is reproducible for late stage melanoma, suggesting that the clonal expansion of cancer-associated CDR3s in the PBMCs might be universal to cancer types and stages. Therefore, we anticipate more clinical efforts to collect PBMC repertoires profiled from early stage cancer patients to elucidate the feasibility of this non-invasive approach for cancer detection, or immune monitoring during cancer therapies.

In summary, we provided a comprehensive analysis to characterize cancer antigens and tumorreactive $\mathrm{T}$ cells. The tool and datasets from this study can be applied to the rapidly generated tumor single-cell sequencing and RNA-seq data to expand the current repertoire of cancerassociated TCRs. Therefore, we anticipate broad utilities of our work for future studies to identify more antigens and biomarkers for cancer immunotherapies. 


\begin{abstract}
Abbreviations
TCGA: The Cancer Genome Atlas; CDR3: Complementarity Determining Region 3; ACC: adenocortical carcinoma; BLCA: bladder carcinoma; BRCA: breast carcinoma; CHOL: cholangiocarcinoma; CESC: cervical squamous carcinoma; COAD: colon adenocarcinoma; DLBC: diffusive large B-cell lymphoma; ESCA: esophageal carcinoma; GBM: glioblastoma multiforme; HNSC: head and neck carcinoma; KICH: kidney chromophobe; KIRC: kidney renal clear cell carcinoma; KIRP: kidney renal papillary cell carcinoma; LAML: acute myeloid leukemia; LGG: lower grade glioma; LIHC: liver hepatocellular carcinoma; LUAD: lung adenocarcinoma; LUSC: lung squamous carcinoma; MESO: mesothelioma; OV: ovarian serous cystadenocarcinoma; PCPG: pheochromocytoma and paraganglioma; PAAD: pancreatic adenocarcinoma; PRAD: prostate adenocarcinoma; READ: rectum adenocarcinoma; SARC: sarcoma; SKCM: skin cutaneous melanoma; STAD: stomach adenocarcinoma; TGCT: testicular germ cell tumor; THCA: thyroid carcinoma; THYM: thymoma; UCEC: uterine corpus endometrial carcinoma; UCS: uterine carsinosarcoma
\end{abstract}

\title{
Acknowledgement
}

This work is supported by CPRIT grant 50C1284401 (BL), Circle of Friends Cancer Center Grant 2018 (BL), and CPRIT grants RR150072 (YXF). We thank Dr. James Brugarolas for helpful discussions during manuscript preparation.

\section{Author contributions}

BL conceived the project, developed the algorithm and performed the computational analysis. LCL designed and performed the in vivo experiment. JZ contributed to algorithm coding. JHC, JFY, XWZ, JQ and AF contributed to data analysis. SS and CW contributed data. BL and LCL wrote the manuscript. BL and YXF supervised the study.

\section{Data and code availability}

iSMART source code is available at https://bitbucket.org/lilab_utsw/ismart/. The CDR3 clustering data is hosted on Firecloud (https://software.broadinstitute.org/firecloud/) and is available upon request under TCGA controlled access.

\section{Conflict of interests}

The authors declare no conflict of interests. 


\section{Methods}

\section{Data resources information}

TCGA level-2 RNA-seq data aligned to hg19 human reference genome by MapSplice ${ }^{61}$ were downloaded from GDC legacy archive (https://portal.gdc.cancer.gov/legacy-archive/). Gene expression data (TPM), mutation annotation files and patient clinical information of the TCGA cohort were downloaded from GDAC broad firehose (https://gdac.broadinstitute.org/). Tumor purity information was downloaded from the Cistrome TIMER website (http://cistrome.org/TIMER/misc/AGPall.zip). TCR repertoire data and patient information for the HCMV cohort, late stage melanoma and early breast cancer samples, were downloaded from AdaptiveBiotechnology immunoSeq Analyzer (https://www.adaptivebiotech.com/). Antigenspecific CDR3 sequence information for benchmarking iSMART were downloaded from VDJdb (https://vdjdb.cdr3.net/). GLIPH software package was accessed from GitHub (https://github.com/immunoengineer/gliph). Single cell gene expression data and matched TCR information were downloaded from GEO database (accession number GSE114724).

\section{Materials and animal model:}

HSFX1 derived 9-mer peptide was synthesized by GenScript; CpG oligonucleotide ODN 1826 was purchased from InvivoGen with catalog number 1826-1; Polyinosinic-polycytidylic acid sodium salt, or Poly (I:C), was ordered from MiliporeSigma with catalog number P1530-25MG. Immunocompetent C57BL/6J and transgenic C57BL/6-Mcph1Tg(HLA-A2.1)1Enge/J mice were obtained from Jackson Laboratory (JAX:000664 and JAX:003475)

\section{iSMART for pairwise CDR3 alignment and clustering}

iSMART takes $M$ complete CDR3 sequences as input, where complete CDR3 region is defined as the last cysteine in the variable gene to the first amino acid in the FGXG motif in the joining gene ${ }^{28}$. iSMART first orders the CDR3s according to their lengths, and then performs pairwise comparisons for every sequence. For CDR3s with different lengths, iSMART allows at most one insertion in the comparison, and imposes a gap penalty (default 6). Alignment scores are calculated based on BLOSUM62 matrix, with individual matched score capped at 4 . The $3^{\text {rd }}$ to $(n-$ $3)^{\text {th }}$ positions of the CDR3s are used for scoring, where $n$ is the CDR3 amino acid sequence length. Pairwise alignment score is normalized by the length of the longer CDR3 sequence ( $n-4$, excluding first and last 2 amino acids). After calculation of the $M$-by- $M$ pairwise scoring matrix, a predefined cutoff value (default 3.5) is applied to filter out all the low scoring comparisons. iSMART then performs a depth-first search on the matrix to identify all the connected CDR3 clusters, and output all the CDR3s with empirical cluster IDs. iSMART is written in Python and the source code is publicly available.

Although iSMART is benchmarked to run without variable gene assignment in this work, it supports the input with variable gene information. In this mode, the pairwise alignment on the CDR3 regions is the same except that iSMART uses the $5^{\text {th }}$ to $(n-3)^{\text {th }}$ positions of the sequence for scoring. As the first 4 amino acids of the CDR3s are mainly determined by the variable gene, we made this change to avoid repeated use of variable gene information. In the pairwise sequence comparison step, the CDR1 and CDR2 regions of two TCRs are also used to calculate alignment scores under the same rules. The total score is scaled to 8, where CDR3 and variable gene contribute equally, and a cutoff value (default 7.5) is used to generate the CDR3 clusters. iSMART in variable gene mode was tested using the 15 antigen benchmark dataset, which is described in section below, and reached a higher specificity of $94.3 \%$ (100 out of 106 clusters have unique antigen assignment) than without variable gene input. 


\section{iSMART and GLIPH performance evaluation}

Both iSMART and GLIPH can predict antigen-specific CDR3 clusters without variable gene information. In this work, we evaluated the performances of both methods using TCRs of known antigen-specificity in VDJdb ${ }^{34}$. We selected 15 9-mer human antigens with balanced number $(\mathrm{K})$ of associated TCR $\beta$ CDR3s $(100<\mathrm{K}<1000)$ (Supplementary Table 1). CDR3s associated with more than one antigens were excluded, resulting in a total of 2,347 unique sequences. Both iSMART and GLIPH were run on this dataset with default parameters.

The command line for iSMART is:

python iSMARTV1.py -f human15aa.txt $-\mathrm{v}$

where $-v$ option is applied to disable the use of variable gene. For GLIPH the command line is: ./gliph-group-discovery.pl --tcr human15aa.txt

Interestingly, although iSMART performs time-consuming pairwise sequence alignments, its computational time (63s) is significantly less than GLIPH (approximately 1 hour) on MacBook Pro with $3.1 \mathrm{GHz}$ Intel core i7 and 16 GB DDR3 memory. Therefore, iSMART has the computational efficiency to scale up for larger TCR repertoire datasets.

As each CDR3 is uniquely linked to one antigen in the benchmark dataset, we defined cluster purity $(p)$ as the number of the most abundant antigen divided by the number of CDR3s in a cluster. We use the percent of completely pure $(p=1)$ clusters as a measure for specificity. To make visualization of the clustering specificity, we computed the cross-antigen classification errors as follows: the 15 -by-15 cross-antigen matrix $(M)$ is initialized by 0 , and for each cluster, let A denote the set of antigens associated with the CDR3s in this cluster, we add 1 to all the entries in $M[\mathrm{~A}, \mathrm{~A}]$. Therefore, if A contains only one antigen, the diagonal values for $M$ will increase by 1 . Otherwise the off-diagonal values will increase by 1 , which are considered as classification errors. We looped through all the clusters and used the final output to plot the heatmaps in Figure 1 (iSMART) and Supplementary Figure 1 (GLIPH).

\section{Non-cancerous public TCR identification}

A critical pre-processing procedure in our analysis is to exclude non-cancerous public TCRs to reduce false positives in our downstream analysis. We used a cohort of non-cancer individuals with TCR repertoire sequencing data available ${ }^{30}$. There are two batches of this cohort, with the first batch containing 666 human cytomegalovirus (HCMV) infected $(n=289)$ or normal individuals. The HCMV infected individuals can be used as control samples for our purposes. The second batch contains 120 individuals. We will use the first batch to remove public TCRs and the second for downstream analysis, to avoid systematic bias. Based on antigen-specificity, we processed two classes of public TCR sequences:

The first class is antigen-specific non-cancerous CDR3s. In our downstream analysis of detection cancer-associated CDR3s in the blood TCR repertoire, we also rely on this HCMV cohort as normal control. Therefore, at this step, to prevent any potential confounders, we used the first batch $(n=666)$ to remove public TCRs. It is known that cancer-specific T cells are also present in healthy individuals in the form of low abundant naïve T cells ${ }^{62}$. Therefore, to prevent false removal of bona fide cancer-specific CDR3s, we restricted our analysis within the top 5,000 most abundant sequences, sufficient to cover all the clones with $\geq 5$ copies that are expected to be effector $\mathrm{T}$ cells. We combined all the sequences as normal CDR3s to be removed in the TCGA data before iSMART 
clustering. The resulting dataset as well as samples used in this analysis are available as Supplementary Dataset 1.

The second class of public sequences are non-antigen specific CDR3s, potentially due to biased $\mathrm{V}(\mathrm{D}) \mathrm{J}$ recombination. We anticipate that the sharing of these sequences between individuals is not affected by the HLA alleles of the carriers. Therefore, we performed 800,000 random sampling of triplets from the pool of 666 TCR repertoire samples satisfying the following criterion: there is no overlap in the HLA alleles in any two individuals in the triplet. For each triplet, we compared the top 5,000 most abundant sequences in each sample and selected those appeared in all three. The resulting 3,470 CDR3 sequences are available as Supplementary Dataset 2.

We removed both classes of public sequences from the 170,516 complete CDR3s and obtained 82,427 non-public sequences for downstream analysis. As the TCR repertoire data in the public domain are mainly $\beta$ chain sequences, currently we do not have enough data to eliminate public $\alpha$ chain CDR3s from the analysis. We will rely on future efforts to sequence more TCR $\alpha$ chain repertoire samples to define public $\alpha$ chain CDR3 sequences.

\section{Analysis of single cell sequencing data}

Post-processed gene expression data in sparse matrix format ( $\mathrm{mtx}$ ) and TCR hypervariable CDR3 sequences with matched cell barcodes were downloaded directly from the GEO database. In total, there are 5 samples from 3 patients, BC09, BC10 and BC11. BC10 has the largest overlap with TCGA-derived CDR3 clusters. For BC10, we selected 1,103 genes with standard deviation $\geq 1$ and performed tSNE analysis on the 4,926 cells using these genes for dimension reduction. This filter is purely for visualization purposes. 2-dimensional scatter plot using tSNE values were generated to visualize the distributions of genes of interest. Based on the locally enriched pattern of 18 clustered cells, we defined a subgroup of 44 cells. For each of the 1,103 genes, we performed Wilcoxon rank sum test between this group and other cells and used Benjamini-Hochberg method to evaluate FDR. These results, including the cell barcodes for the selected group, are available in Supplementary Table 3. ZNF683 expression levels in the TCGA samples were split into two groups by the median level. Survival analysis for ZNF683 was performed using Cox proportional hazard model on the binary variable corrected for patient age.

We performed cell trajectory analysis for selected clonotypes in the breast cancer samples. For sample BC10, we selected 418 cells with CDR3 sequences found in the CD8+ subgroup identified in the tSNE plot, and used R package monocle ${ }^{48}$ to perform cell ordering by pseudotime. As the direction of pseudotime is arbitrary, we used representative biomarkers for $\mathrm{T}$ cell activation to determine the beginning of the trajectory, and identified the $\mathrm{T}_{\text {pre }}$ population. The $\mathrm{T}_{\mathrm{rm}}$ clusters were then selected at the end of the trajectory. Spearman's correlation between each gene expression level and pseudotime was calculated, and we selected important biomarkers for cell identity (IL7R, TCF7, CCR7), cytotoxicity (GZMB, PRF1, IFNG), exhaustion (PD-1, LAG3, TIM-3), resident memory signature (SELL, KLRG1, CD103) and metabolic status (OXPHOS genes). For BC11, we first merged the two biological replicates into one dataset and selected 31 cells with IL7R $\leq 1, T C F 7 \leq 1, G Z M B \geq 5$, ZNF683 $\geq 5$ and CD103 $\geq 10$ as tissue resident T cells, and used all the 728 cells sharing the same CDR3s with these 31 cells to perform the pseudotime trajectory analysis. These cells in total come from 11 clonotypes, but for the individual clonotype evolution analysis, we removed two clonotypes with $n=1$ and showed the remaining 9 in Supplementary Figure 8c. We did not identify any cell using the same selection criteria for tissue resident memory cells for sample BC09. 


\section{Gene expression analysis}

We performed a genome-wide correlative analysis to identify genes associated with counts $(K)$ of clustered CDR3s in each individual (Supplementary Figure 4). We first selected 15 cancer types with sufficient sample size $(\geq 100)$. For each cancer, we calculated partial Spearman's correlation between $K$ and the expression level for each gene. Tumor purity is corrected in this analysis as it is expected to impact gene expression profiles ${ }^{63}$ and is correlated to $\mathrm{T}$ cell infiltration. False discovery rate is estimated using Benjamini-Hochberg procedure for all the $\mathrm{p}$ values pooled.

We also performed differential gene expression analysis to identify novel cancer associated antigens (Figure 4). First, we selected 120 clusters with CDR3 length $20 \geq \mathrm{L} \geq 13$ and with $\geq 10$ sequences. For each cluster, we performed one-tailed Wilcoxon rank sum test for each gene between clustered and non-clustered individuals from all cancers, pooled all the p values and estimated FDR using Benjamini-Hochberg correction. This step selected 3,524 significant results (FDR $<0.05$ and fold change $\geq 10$ ), including 1,409 unique genes spanning 115 clusters. Fold change was calculated for each cluster, as the median expression value of the samples in the CDR3 cluster divided by that of those not in the cluster. If the denominator is zero, we used an arbitrarily small number $10^{-13}$. Of all the protein coding genes, HSFX1 has the top significant value, and is associated with clusters 1724 and 1767 . We performed a second differential gene expression analysis to visualize the top highly expressed genes, by combining samples in the two clusters.

\section{Vaccination of naïve and transgenic mice and ELISPOT assay}

C57BL/6J and C57BL/6J-HLA-A2.1Tg mice were purchased from the Jackson Laboratory. All mice were maintained under specific pathogen-free conditions at UT Southwestern Medical Center. 10 $\mu \mathrm{g}$ of VMF or VRF peptide was mixed with $50 \mu \mathrm{g}$ ODN1826 and $100 \mu \mathrm{g}$ poly (I:C) in $100 \mu \mathrm{l}$ PBS and then subcutaneously injected to the mouse on day 0 and day 14 . Single cell suspensions were prepared on day 18 post first vaccination. Splenocytes were seeded at $4^{*} 10^{5}$ per well and stimulated with either $10 \mu \mathrm{g}$ peptide or PMA + Ionomycin for 36 hours. ELISPOT assay was performed using an IFN- $\gamma$ ELISPOT assay kit (BD Biosciences) according to the manufacturer's instruction. Spots were enumerated by ImmunoSpot Analyzer (CTL).

\section{Analysis of missense and frameshift mutations}

In total we analyzed 920,483 somatic missense mutations from 7,046 TCGA samples with whole exome sequencing data. Mutations occurred in fewer than 3 individuals were excluded, resulting in 6,136 mutations across 5,774 individuals. For each mutation, we estimated its co-occurrence with each of the 671 clusters with CDR3 length $20 \geq \mathrm{L} \geq 14$ and with $\geq 3$ sequences. The length cutoffs were applied to minimize the inclusion of public TCRs, or potentially incorrect CDR3 assemblies. The cluster size cut-off was applied to select those with potentially sufficient statistical power. For each comparison, Fisher's exact test was performed to estimate a p value (p), only when there is an overlap between the mutation carriers, and those in the CDR3 cluster. It is clear that the $\mathrm{p}$ values generated in this approach were inflated due to the selection criterion. Therefore, we conducted a permutation analysis to evaluate the real significance levels and correct for multiple hypothesis testing.

We initialized loop counter $n=0$ and started iterations. Each time, we randomly sampled one CDR3 cluster (denoted as $C$ ). Let $\mathrm{n}_{\mathrm{c}}$ denote the number of individuals in $C$, we randomly sampled $\mathrm{n}_{\mathrm{c}}$ unique IDs from the pool of 5,774 individuals to replace $C$. We then randomly sampled one mutation, and estimated the co-occurrence between $C$ and the mutation carriers. If there was no overlap, the loop started over again without changing $n$. If an overlap existed, we estimated the $p$ 
value using Fisher's exact test, and $n$ became $n+1$. The loop stopped at $n=50,000$. $P$ values $\left(P_{0}\right)$ produced from this analysis were used as null distribution to evaluate the corrected significance levels and FDR. Specifically, for each $\mathrm{p}$, we calculated the significance level $\mathrm{p}^{\prime}=\left(\right.$ number of $\mathrm{P}_{0}$ smaller than p)/50,000. FDR was then estimated using Benjamini-Hochberg procedure on p'.

Similarly, we analyzed 53,491 frameshift indels and kept 1,225 ones occurring for $\geq 3$ times. These mutations distributed across 2,810 individuals. We used the same set of CDR3 clusters to study the co-occurrence patterns between mutation and CDR3s, and used the same permutation strategy to evaluate statistical significance and FDR. If one CDR3 cluster was associated with more than one passed-FDR mutations (including indels), we used the most significant one in our downstream analysis. For both types of variants, we selected mutations with FDR $\leq 0.05$ and odds ratio from the Fisher's exact test $\geq 1000$. The cut-off in the odds ratio was applied to select highly specific enrichment of mutations in the CDR3 clustered individuals.

\section{HLA allele binding prediction}

All the HLA allele binding predictions in this work were performed using either NetMHC or NetMHCpan online server. We implemented NetMHCpan for less common HLA alleles not covered in NetMHC. For missense mutations, the input peptide is a 17-mer with mutated amino acid in the middle. For frameshift mutations, we included 8-mer before and all the amino acid sequence after the mutation locus. For cancer-associated antigens, we downloaded the complete protein sequence from Uniprot (www.uniprot.org), and input the fasta file to NetMHC/NetMHCpan server. Biding of the control peptide VRF for in vivo validation to HLA-A*02:01 was predicted using NetMHC server. Default rank cut-offs were applied to define weak $(\leq 2)$ or strong binders $(\leq 0.5)$.

\section{Prediction of cancer disease status}

In this analysis, we compared 3 TCR repertoire datasets from different studies, including pre/post anti-CTLA4 treatment late stage melanoma (melanoma), early breast cancer (breast cancer), and HCMV cohort (HCMV) as normal control. To avoid systematic bias after public TCR removal, we used second batch $(n=120)$ of the HCMV cohort in this analysis. Direct comparison between different study cohorts will be biased towards sequencing depth and the amount of lymphocytes captured for sequencing. Therefore, we conducted a downsampling procedure to ensure the comparability across cohorts. The targeted capture protocol applied for TCR repertoire sequencing allowed one read to completely cover the whole CDR3 region. Therefore, read count is used to estimate clonal abundance from the Adaptive Biotechnology immunoSEQ Analyzer. We first calculate the size for each TCR-seq library $(\mathrm{N})$, which is the summation of the read counts $(\mathrm{m})$ for all the CDR3 calls. A combined vector of CDR3s with length $\mathrm{N}$ was made, with each CDR3 sequence $i$ repeated by $m_{i}$ times, where $m_{i}$ is the read count for CDR3 sequence $i$. For the melanoma cohort, we used all the cancer samples $(n=21$ for either pre- or post- treatment), and randomly sampled 100 individuals with replacement as normal control. For each of the 121 samples, we downsampled the library to $\mathrm{K}=100,000$ reads, each read being a CDR3 amino acid sequence. The read count $\left(m^{\prime}\right)$ for each unique CDR3 was then calculated. For each sample, CDR3s with identical sequence to one of the cancer-associated CDR3s were selected, and the summation of $m^{\prime}$ for all the selected CDR3s was used as predictor for cancer status. For breast cancer cohort, same downsampling strategy was applied, except that we used K=30,000 for PBMC and 10,000 for TIL samples.

\section{Statistical Analysis}


Statistical analyses were performed using R statistical programming language ${ }^{64}$. Survival analysis was implemented using Cox proportional hazard model in R package survival. Receiver operator curves and area under curve calculations were performed with R package AUC. tSNE plots for single cell analysis were generated using Rtsne $e^{65}$. Single cell pseudotime trajectory analysis was performed using cellrangerRkit and monocle ${ }^{48}$. The statistical significance for Figure 5c was estimated separately. The genotype frequency for $A^{*} 02: 01$ in the TCGA cohort is 0.417 , and the frequencies for $C^{*}$ 07:01 and $C^{*}$ 07:02 are smaller. We used $A^{*} 02: 01$ frequency to estimate a conservative $p$ value: the probability of observing 9 individuals carrying $A^{*} 02: 01$ is $0.417^{9}=0.00038$. Therefore, the $\mathrm{p}$ value for observing the configuration in Figure $5 \mathrm{c}$ is significant. Similarly, the most abundant allele type in Supplementary Figure 13c is $C^{*} 07: 01$, with genotype frequency 0.253 , and the $p$ value for this configuration is $\leq 0.253^{5}=0.0010$. Two-way ANOVA test for comparing different treatment groups of vaccinated mice was performed using commercial software GraphPad Prism. 
a

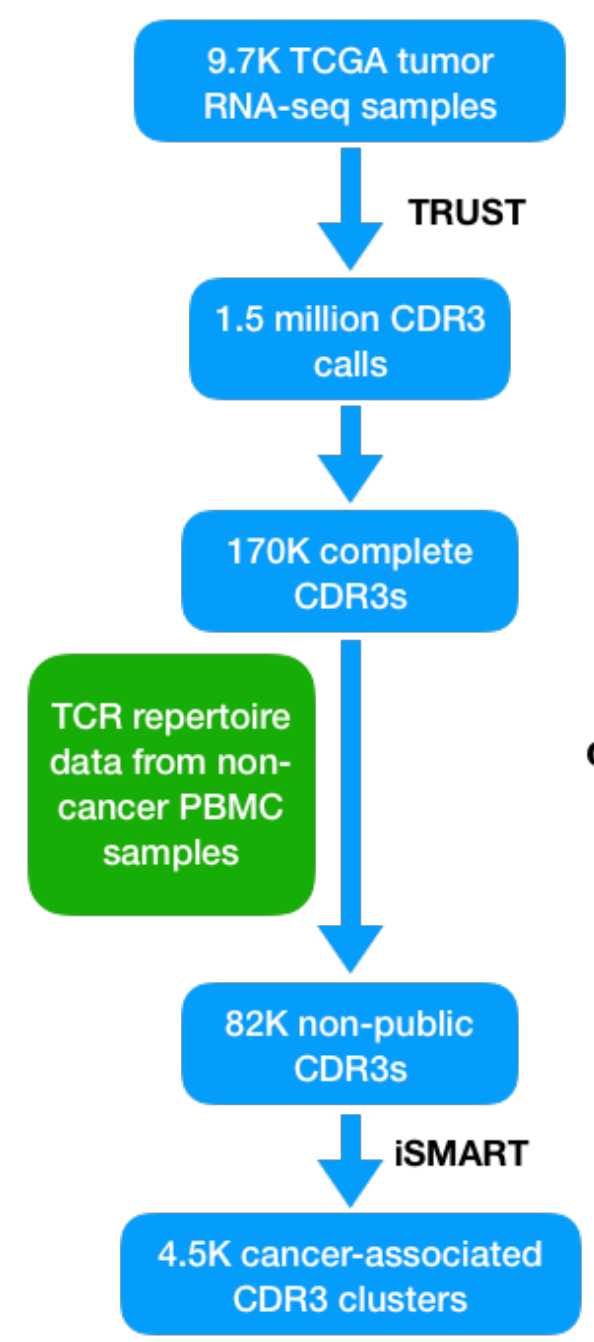

b

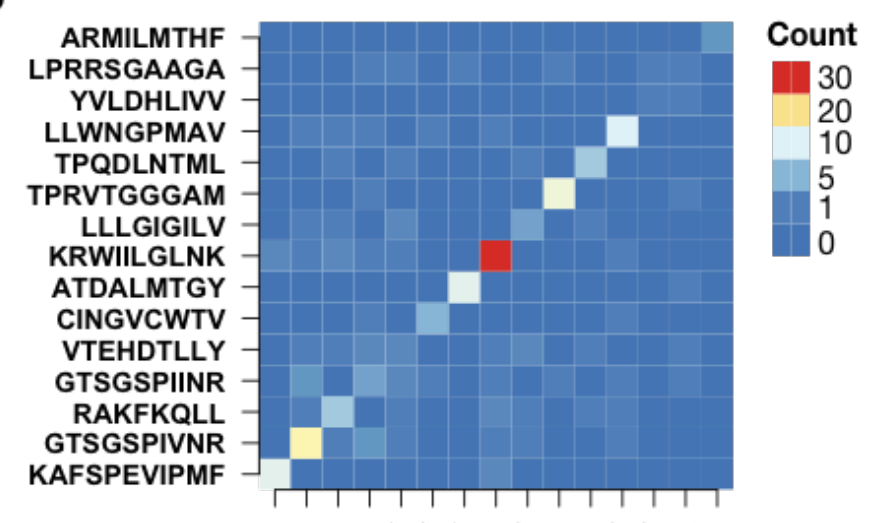

C

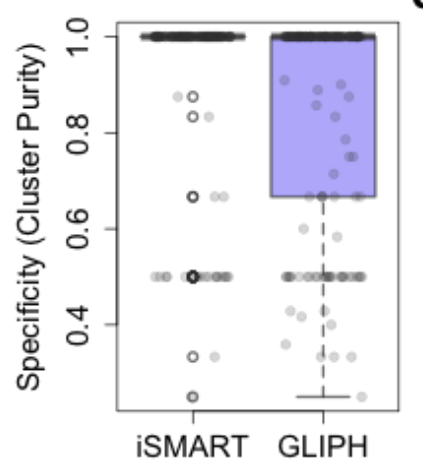

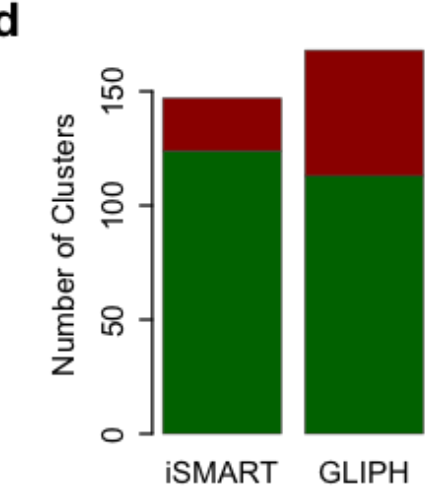

Impure Cluster
Pure Cluster

Figure 1. Methodology summary and performance evaluation for iSMART. a) Flowchart illustrating the analytical procedures carried out in this work to generate CDR3 clusters using TCR receptor sequences extracted from the tumor RNA-seq data. b) Heatmap representation for crossantigen classification errors for iSMART. Each entry in the off-diagonal matrix is an integer representing the number of CDR3 groups showing co-clustering of the two antigens. Diagonal entries recorded the number of clusters with at least 2 CDR3s assigned to the corresponding antigen. c) Specificity comparison between iSMART and GLIPH. Cluster purities were displayed with boxplots, where $84.3 \%$ and $67.3 \%$ clusters have purity equal to 1 for iSMART and GLIPH respectively. d) Barplots showing the number of pure clusters for the two methods as a sensitivity measure to detect antigen-specific clusters. 
a
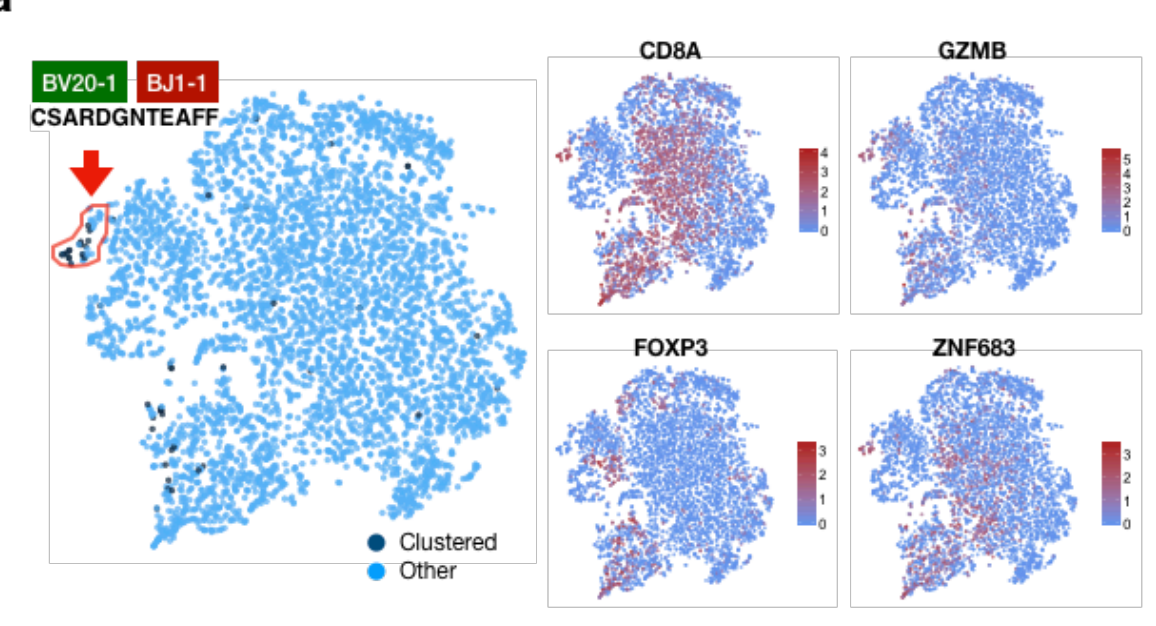

C

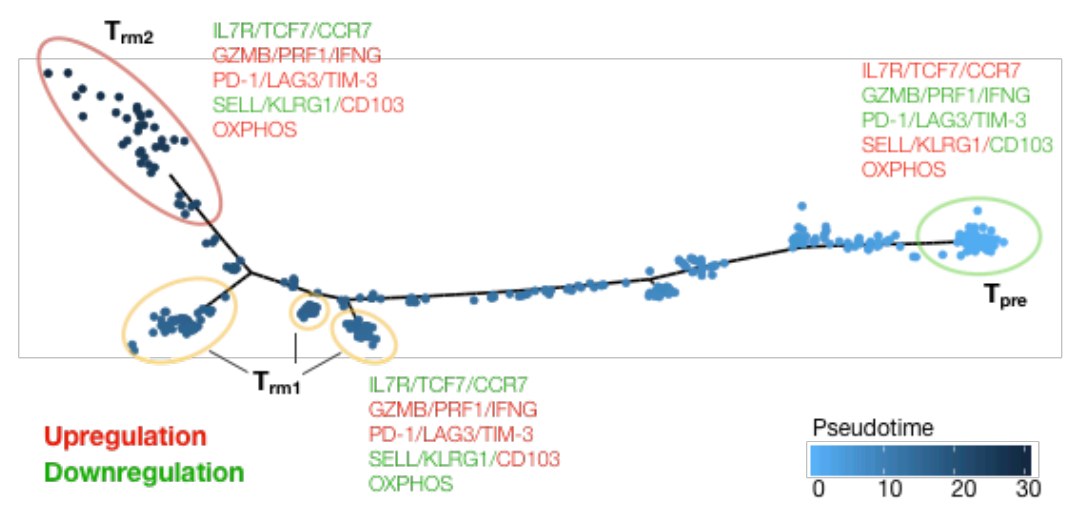

b
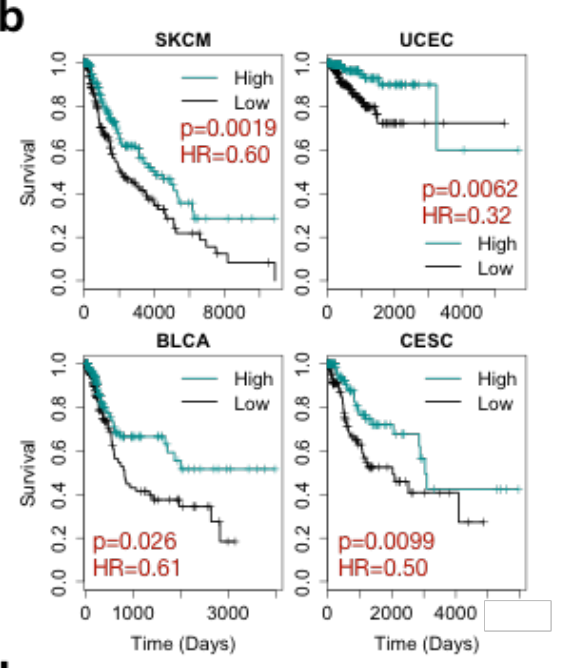

d

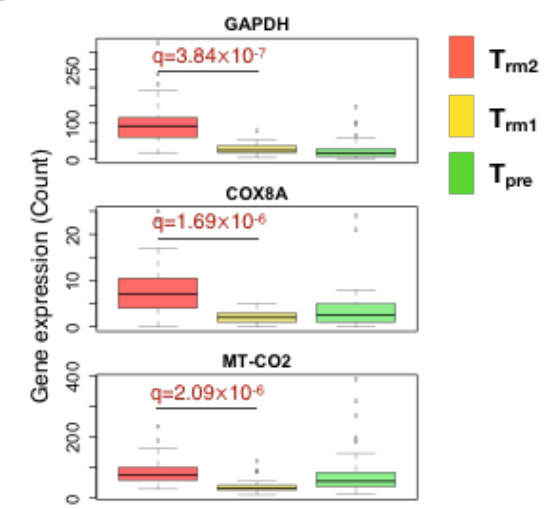

Figure 2. iSMART-clustered clonotypes showing tissue-resident memory phenotype. a) tSNE plots showing the distributions for clustered clonotypes in the TIL population (left), and the expression levels of selected putative markers for cell identity (CD8A/FOXP3/ZNF683) or function $(G Z M B)$ (right four panels). All selected markers passed FDR=0.05. Color legends for gene expression were in log scale. b) Kaplan-Meier curves for four TCGA cancers showing the survival benefit for ZNF683 high expression. For each cancer, median value was applied to define high or low groups. Statistical significance and hazard ratio were evaluated using Cox proportional hazard model. c) Pseudotime trajectory plot illustrating the inferred evolutionary path. Cell clusters located on the beginning or end of the trajectory were manually selected. Representative markers significantly correlated (Spearman's correlation test, FDR $<0.05$ ) with pseudotime inference were labeled for each cluster, with red for negative (high in $\mathrm{T}_{\mathrm{pre}}$ ) and green for positive (high in $\mathrm{T}_{\mathrm{rm}}$ ) correlations. d) Boxplots showing the distributions for selective OXPHOS genes in the three cell clusters shown in c). Statistical significance for differential gene expression between $T_{\mathrm{rm} 1}$ and $\mathrm{T}_{\mathrm{rm} 2}$ was evaluated using Wilcoxon rank sum test, with FDR corrected by Benjamini-Hochberg method. 
a

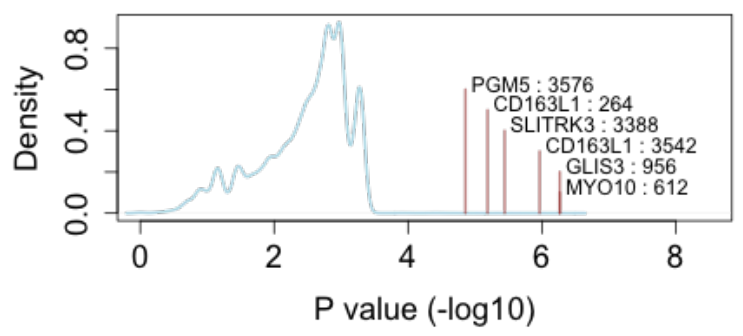

C

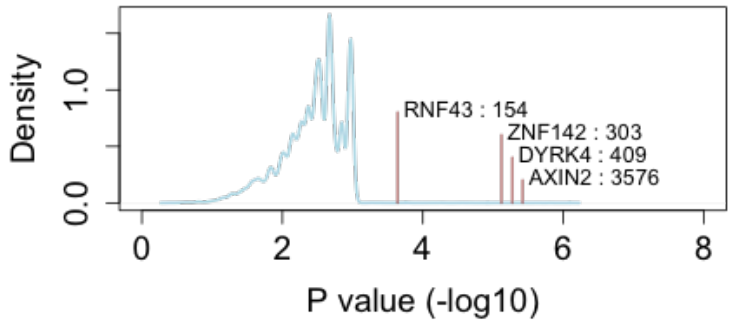

b

\begin{tabular}{|l|c|c|c|}
\hline \multicolumn{1}{|c|}{ Peptide } & HLA allele & Rank & Predicted binder \\
\hline GLIS3: S47L & & & \\
\hline TLSPTMASL & $A^{*} 02: 01$ & 0.22 & SB \\
\hline TLSPTMASL & $\mathrm{C}^{*} 15: 02$ & 0.60 & WB \\
\hline SLITRK3: E968K & & & \\
\hline KVLEKTTYR & $\mathrm{A}^{*} 02: 01$ & 0.046 & SB \\
\hline TKPDYLKVL & $\mathrm{C}^{*} 06: 02$ & 1.34 & WB \\
\hline TKPDYLKVL & $\mathrm{C}^{*} 07: 02$ & 1.15 & WB \\
\hline
\end{tabular}

e

\begin{tabular}{|c|c|c|c|}
\hline Peptide & HLA allele & Rank & Predicted Binder \\
\hline PAPPPVPTC & $C^{*} 12: 03$ & 2.0 & WB \\
\hline TPAPPPVPT & $B^{*} 35: 03$ & 0.50 & SB \\
\hline
\end{tabular}

d

TCGA-CG-4305 AXIN2 deletion at chr17:63532585

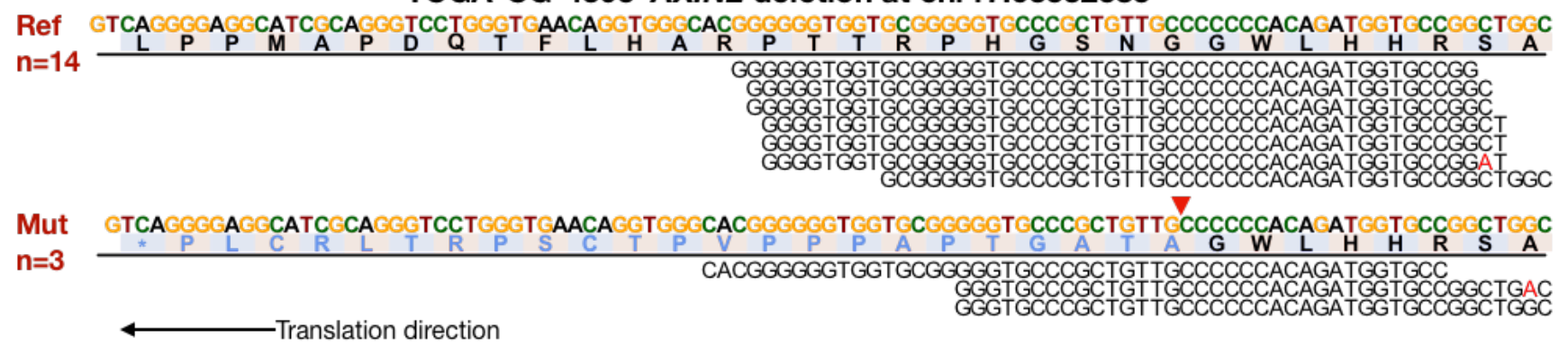

Figure 3. Prediction of neoantigens derived from missense somatic mutations and

frameshift indels. a) Probability density plot for the null distribution of $p$ values testing the significance of co-occurrence between CDR3 clusters and recurrent missense mutations. Significant mutations were marked in the plot with vertical lines and text labels. The numbers after each gene symbol are CDR3 cluster IDs. b) Table showing the NetMHC predicted binder peptide sequences, related HLA alleles and binding strength classification. c) Density plot for the $p$ values from the analysis for frameshift indels. d) Reads pileup plot for reference and mutated alleles in the site with frameshift deletion in gene AXIN2. Numbers of total reads for either reference or mutated alleles were presented in the text box on the left. The gene is reversely translated and a stop codon $\left({ }^{*}\right)$ is generated 23 amino acids downstream of the site of deletion (red arrow). Mismatches were labeled with red color. e) NetMHC predictions for peptide binders generated from the frameshift deletion of AXIN2. 
a
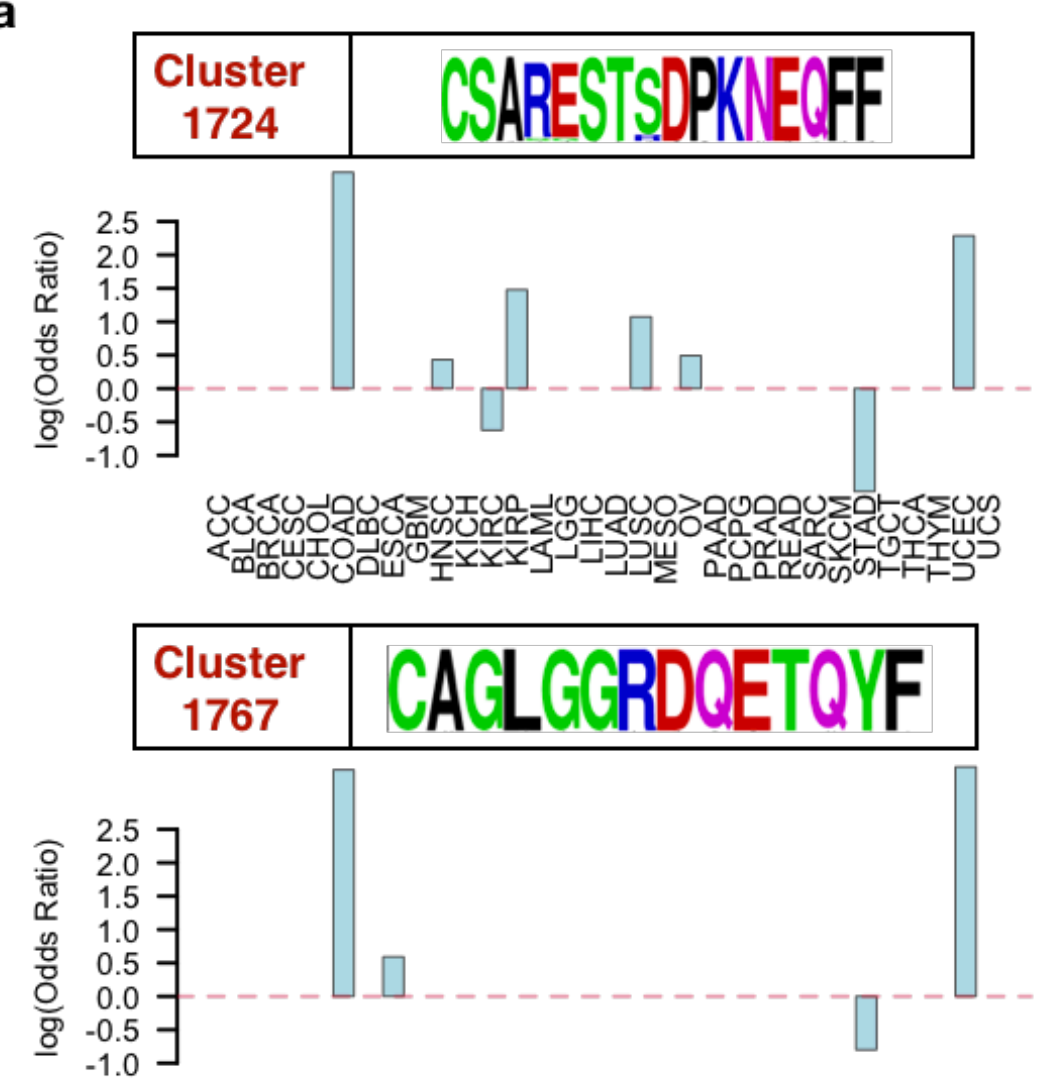

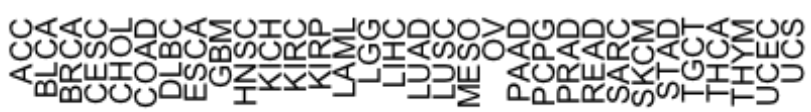

b

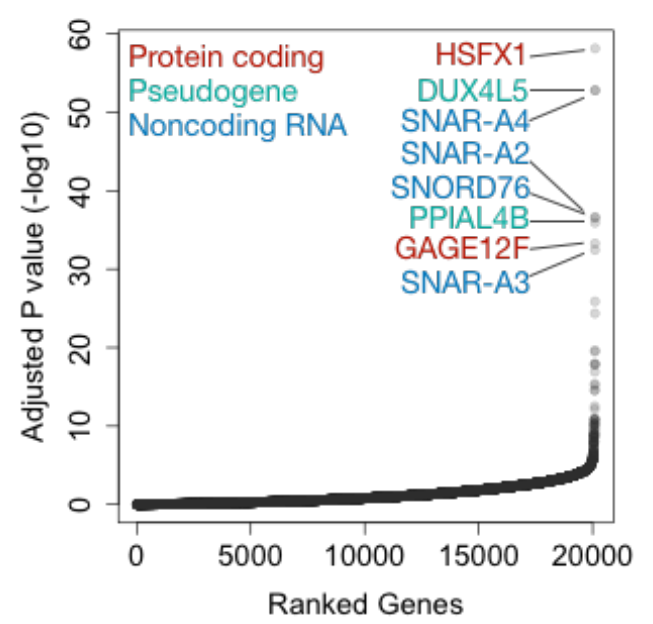

C

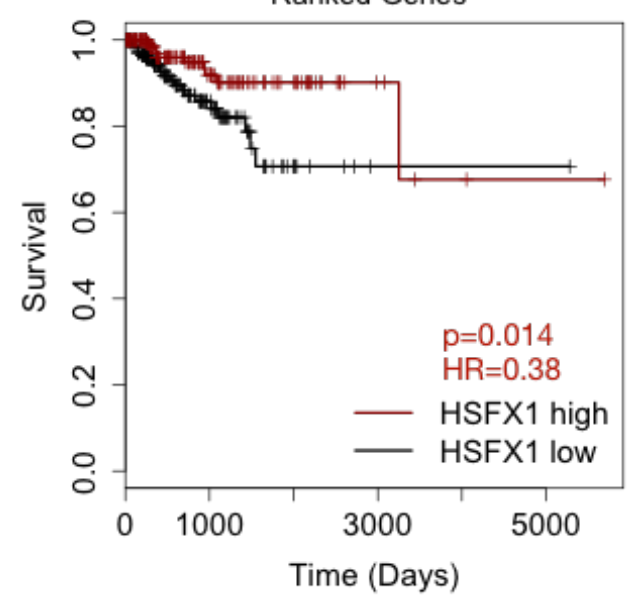

Figure 4. Identification of HSFX1 as a candidate cancer-associated antigen. a) Selective enrichments in colon and endometrial cancers of samples in CDR3 clusters 1724 and 1767. CDR3 amino acid conservation patterns were displayed in the upper panel for each barplot. b) Genes ranked by $\mathrm{p}$ values from differential gene expression analysis, with top hits labeled in colored texts. HSFX1 has the most significant $p$ value among all the genes. Statistical significance was evaluated using Wilcoxon rank sum test with FDR correction. c) Kaplan-Meier survival curves for endometrial cancer patients with or without HSFX1 expression, separated by median expression value. Statistical significance and hazard ratios for HSFX1 levels were estimated using Cox proportional hazard model on binary input of HSFX1 groups, corrected for patient age. 


\section{a}

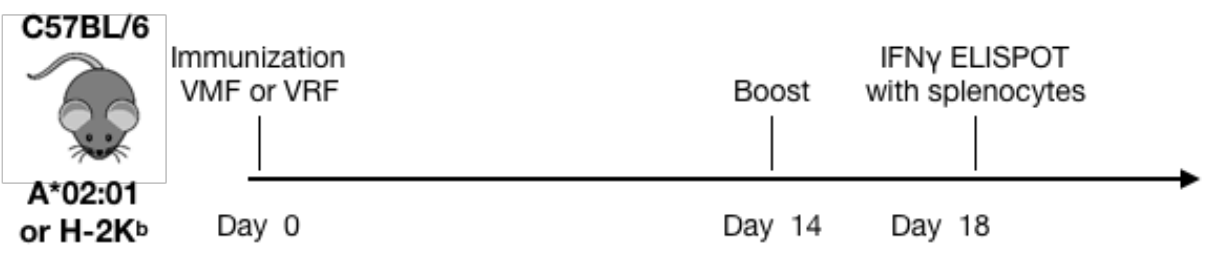

b

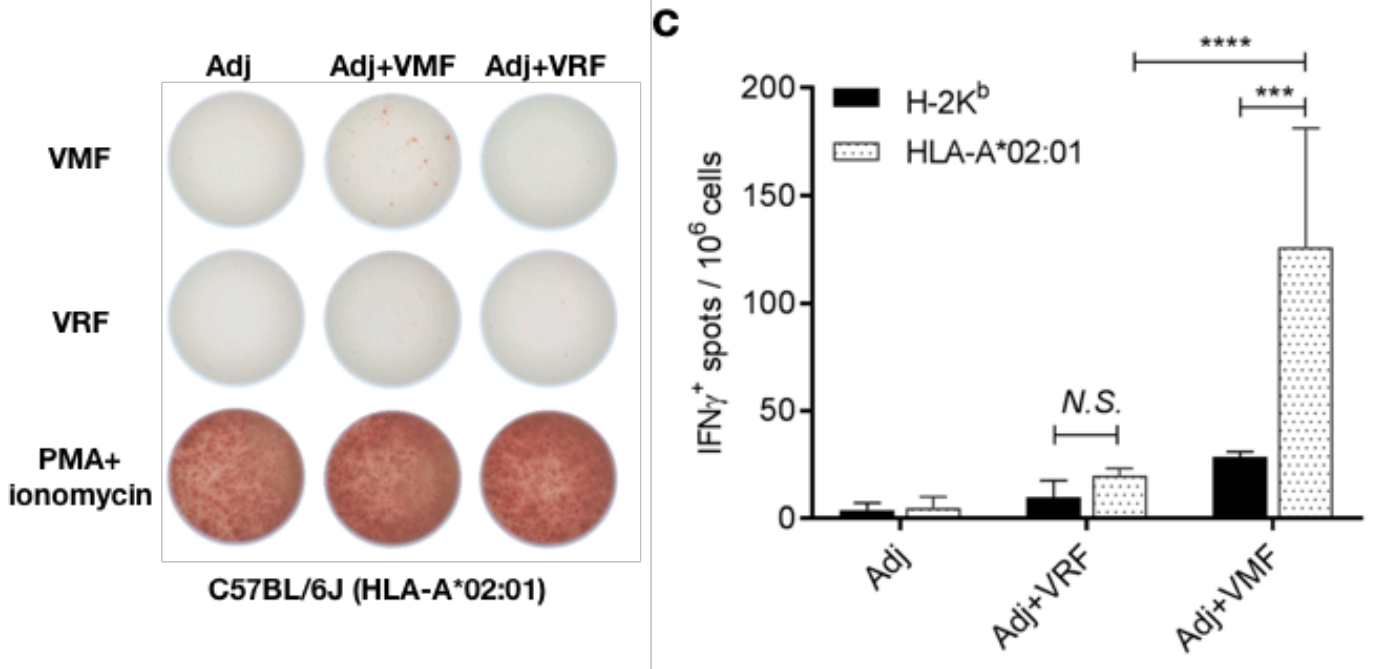

Figure 5. Immunogenicity of the 9-mer peptide derived from HSFX1 protein in HLA-A*02:01 transgenic mice. a) HLA- $A^{*}$ 02:01 transgenic mice (female, $n=4$ ) were subcutaneously immunized

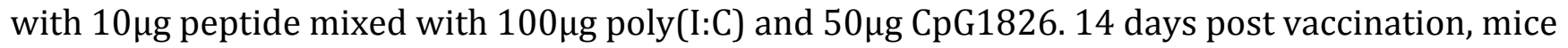
were boosted with the same vaccine. 4 days later, splenocytes were isolated for IFN $\gamma$ ELISPOT assay. Representative results showed IFN $\gamma$ secreting cells from indicated groups (b). Column texts labeled the 3 treatment groups, where the T cells were collected. Row texts labeled simulants used in the ELISPOT assay. Significant difference of antigen-specific T cell response from the control peptide was observed (c). Data are expressed as the means \pm SD, representative results from two independent experiments are shown. Statistics analysis was performed by Two-way ANOVA. ${ }^{* * *}, \mathrm{P}$ $<0.001 ;{ }^{* * * *}, \mathrm{P}<0.0001$. Adj is short for adjuvant injected during vaccination. VMF: antigen peptide VMFPHLPAL; VRF: control peptide VRFPHLPAL; PMA+ionomycin: standard positive control. 
a

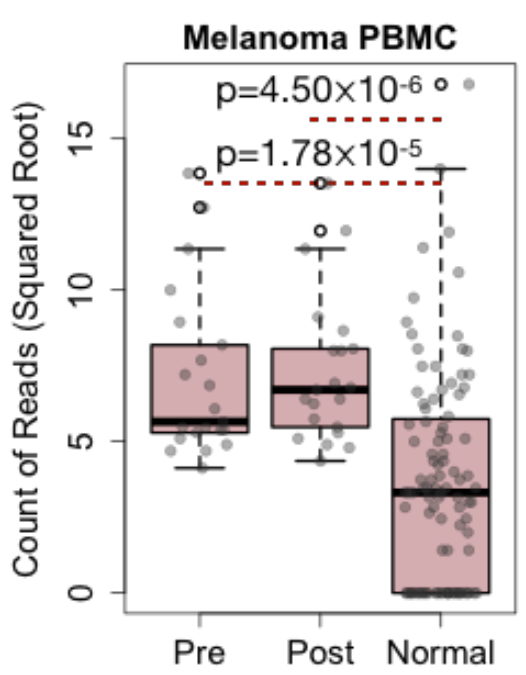

C

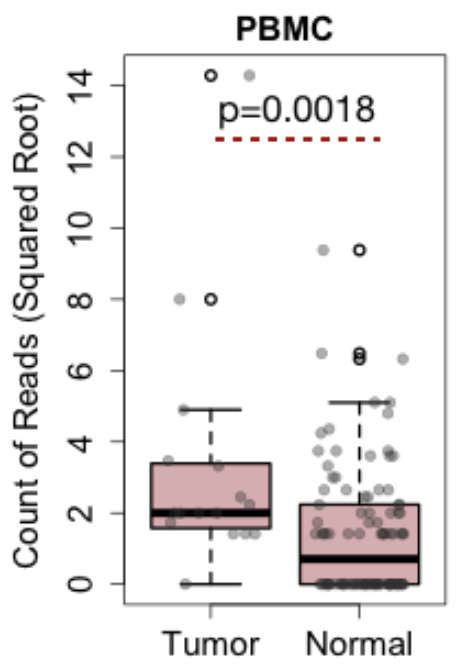

d b
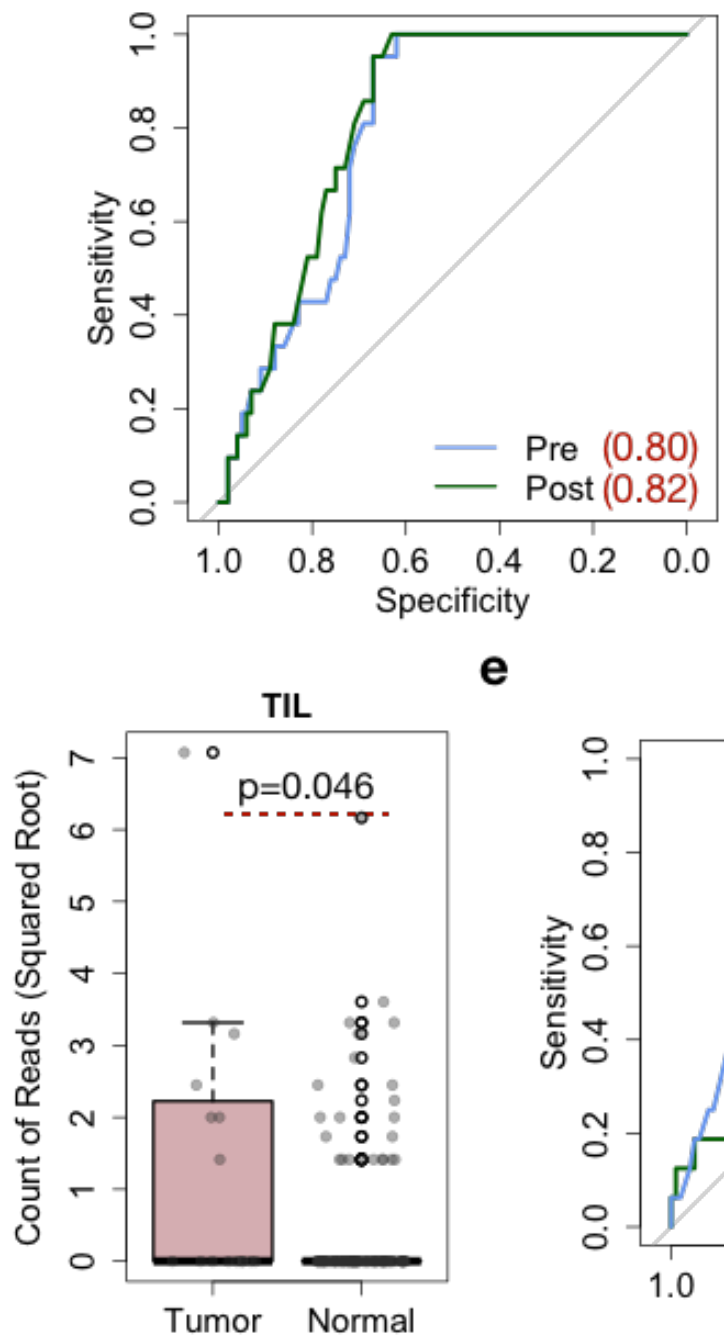

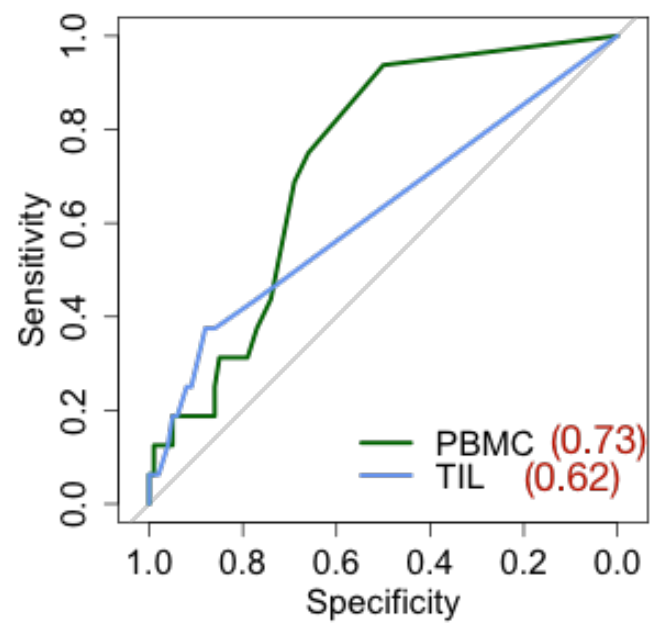

Figure 6. Prediction of late and early stage cancers using cancer-associated CDR3s. a)

Boxplot showing the distributions of the read counts for cancer-associated CDR3s for pre- or postanti-CTLA4 treatment late-stage melanoma and normal control samples. TCR repertoire data from all the samples were derived from PBMCs. b) ROC curves for using CDR3 read count as a predictor for late stage melanoma. Numbers in the figure legend are area under curve (AUC) values. c-d) Cancer-associated CDR3 read count distributions for early stage breast cancers comparing to normal samples, with cancer samples being PBMC (c) or TIL (d). e) ROC curves for using the abundance of cancer-associated CDR3s PBMC or TIL samples as predictors for early breast cancer onset. AUC values were shown in the legend. Statistical significance was evaluated using Wilcoxon rank sum test between labeled groups. 


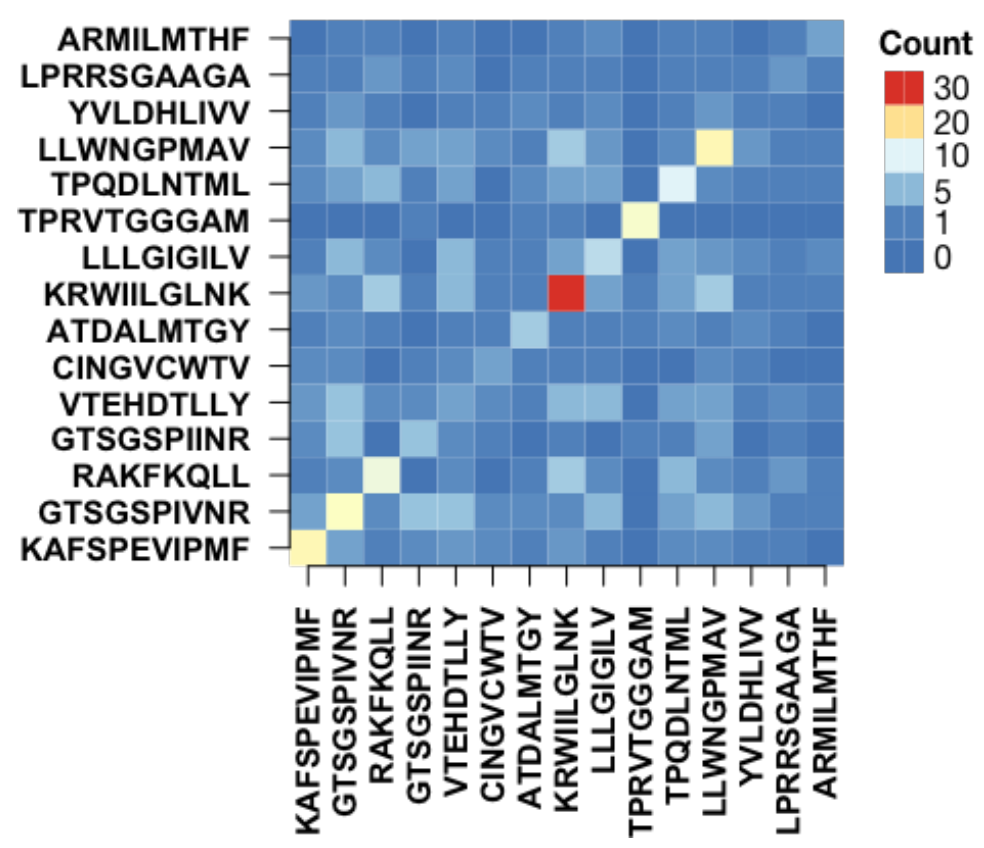

Supplementary Figure 1. Cluster antigen-specificity analysis for GLIPH. Heatmap showing the cross-antigen classification errors from GLIPH predicted CDR3 clusters using the same benchmark dataset of 15 antigens. Same analysis was performed as described in Figure 1b. 
a

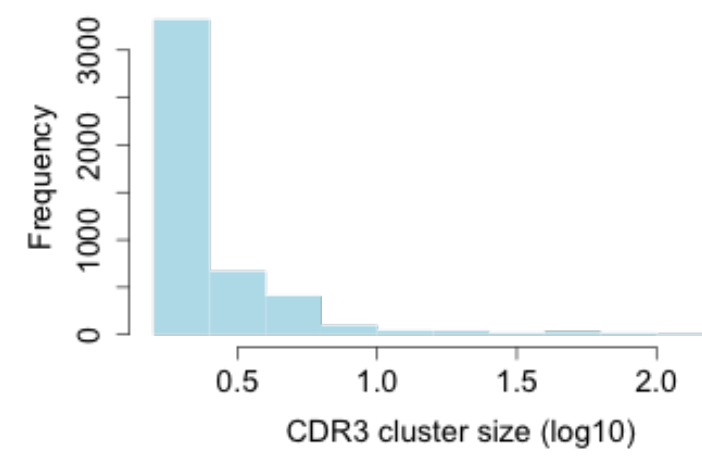

b

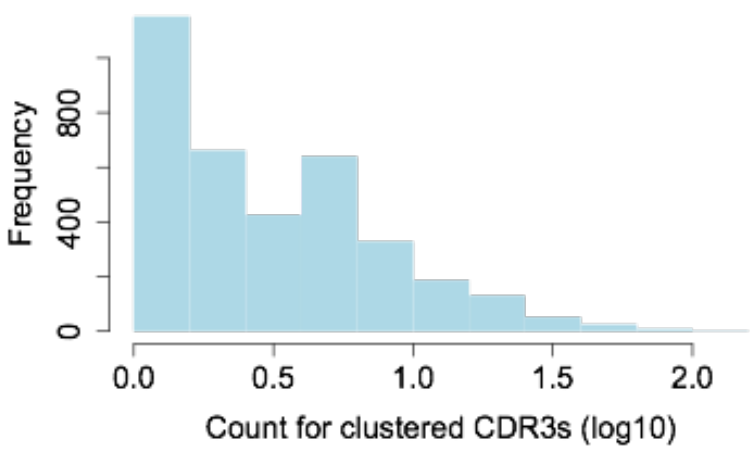

Supplementary Figure 2. Summary of iSMART identified CDR3 clusters. a) Histogram of CDR3 cluster size distribution. b) Lengths distributions for clustered and non-clustered CDR3 amino acid sequences. c) Distribution of the counts for clustered CDR3s carried by each individual in the analysis. 
a

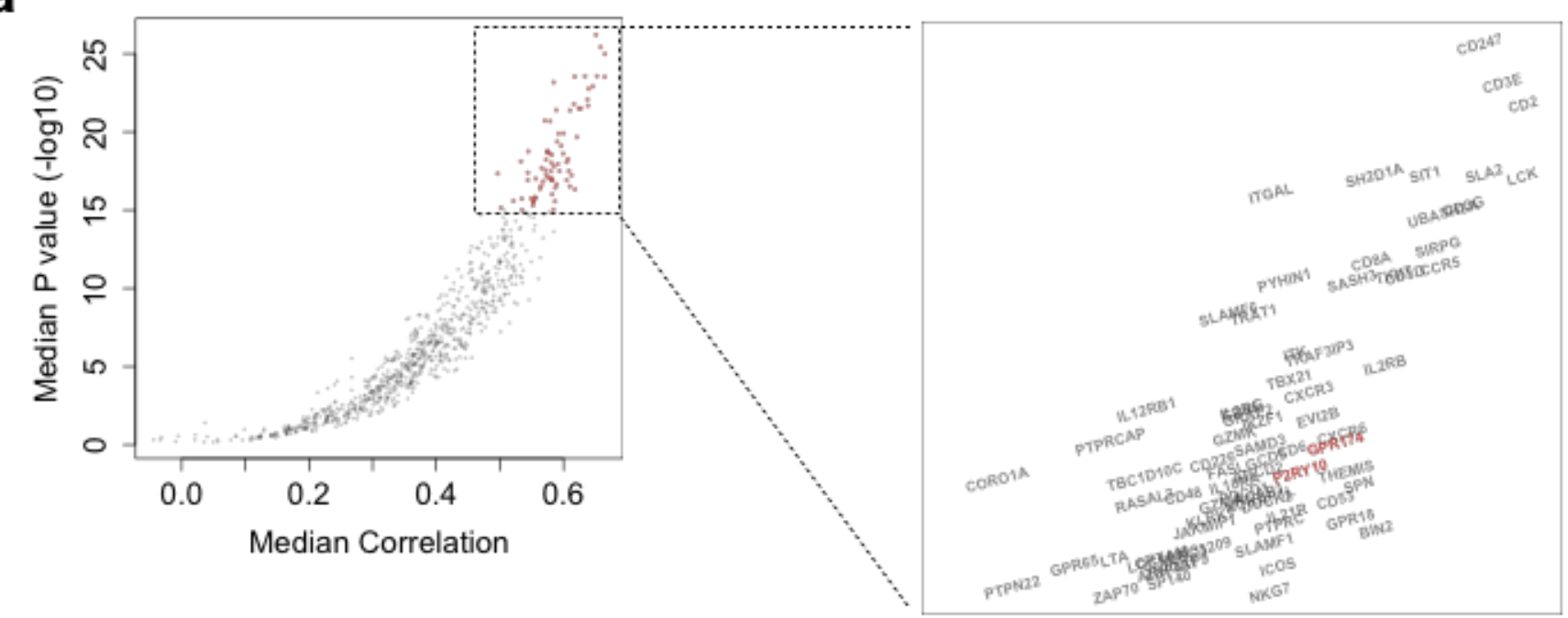

b

\begin{tabular}{|c|c|c|c|c|c|}
\hline Gene Set Name & $\begin{array}{l}\text { \# Genes in } \\
\text { Gene Set (K) }\end{array}$ & $\begin{array}{l}\text { \# Genes in } \\
\text { Overlap (k) }\end{array}$ & $\mathbf{k} / \mathbf{K}$ & p-value & FDR q-value \\
\hline IMMUNE SYSTEM PROCESS & 1984 & 266 & 0.1341 & $1.24 \mathrm{E}-229$ & $7.31 \mathrm{E}-226$ \\
\hline IMMUNE RESPONSE & 1100 & 199 & 0.1809 & $2.13 E-191$ & $6.30 \mathrm{E}-188$ \\
\hline $\begin{array}{l}\text { REGULATION OF IMMUNE } \\
\text { SYSTEM PROCESS }\end{array}$ & 1403 & 208 & 0.1483 & $4.24 \mathrm{E}-182$ & 8.37E-179 \\
\hline $\begin{array}{l}\text { REGULATION OF IMMUNE } \\
\text { RESPONSE }\end{array}$ & 858 & 159 & 0.1853 & $1.24 \mathrm{E}-151$ & $1.84 \mathrm{E}-148$ \\
\hline $\begin{array}{l}\text { POSITIVE REGULATION OF } \\
\text { IMMUNE SYSTEM PROCESS }\end{array}$ & 867 & 152 & 0.1753 & $1.32 \mathrm{E}-140$ & $1.56 \mathrm{E}-137$ \\
\hline DEFENSE RESPONSE & 1231 & 150 & 0.1219 & $3.05 E-114$ & $3.01 \mathrm{E}-111$ \\
\hline LEUKOCYTE ACTIVATION & 414 & 105 & 0.2536 & $1.58 \mathrm{E}-113$ & $1.33 \mathrm{E}-110$ \\
\hline CELL ACTIVATION & 568 & 113 & 0.1989 & $1.97 \mathrm{E}-109$ & $1.46 \mathrm{E}-106$ \\
\hline $\begin{array}{l}\text { REGULATION OF CELL } \\
\text { ACTIVATION }\end{array}$ & 484 & 105 & 0.2169 & $1.12 \mathrm{E}-105$ & $7.39 \mathrm{E}-103$ \\
\hline LYMPHOCYTE ACTIVATION & 342 & 93 & 0.2719 & $2.14 \mathrm{E}-103$ & $1.27 \mathrm{E}-100$ \\
\hline
\end{tabular}

Supplementary Figure 3. Clustered CDR3s as an indicator for activated T cells. a) Volcano plot for genes positively correlated with number of clustered CDR3s. Median values across different cancer types for each gene were calculated for both $p$ value and partial Spearman's correlation with tumor purity correction. Top genes $\left(p \leq 10^{-15}\right)$ were zoomed in for visualization. Genes related to negative regulation for Treg cells were highlighted with dark red color. b) GeneOntology enrichment analysis was performed for the top 500 genes and the top 10 pathways were displayed. Highlighted pathways were related to immune cell activation. 


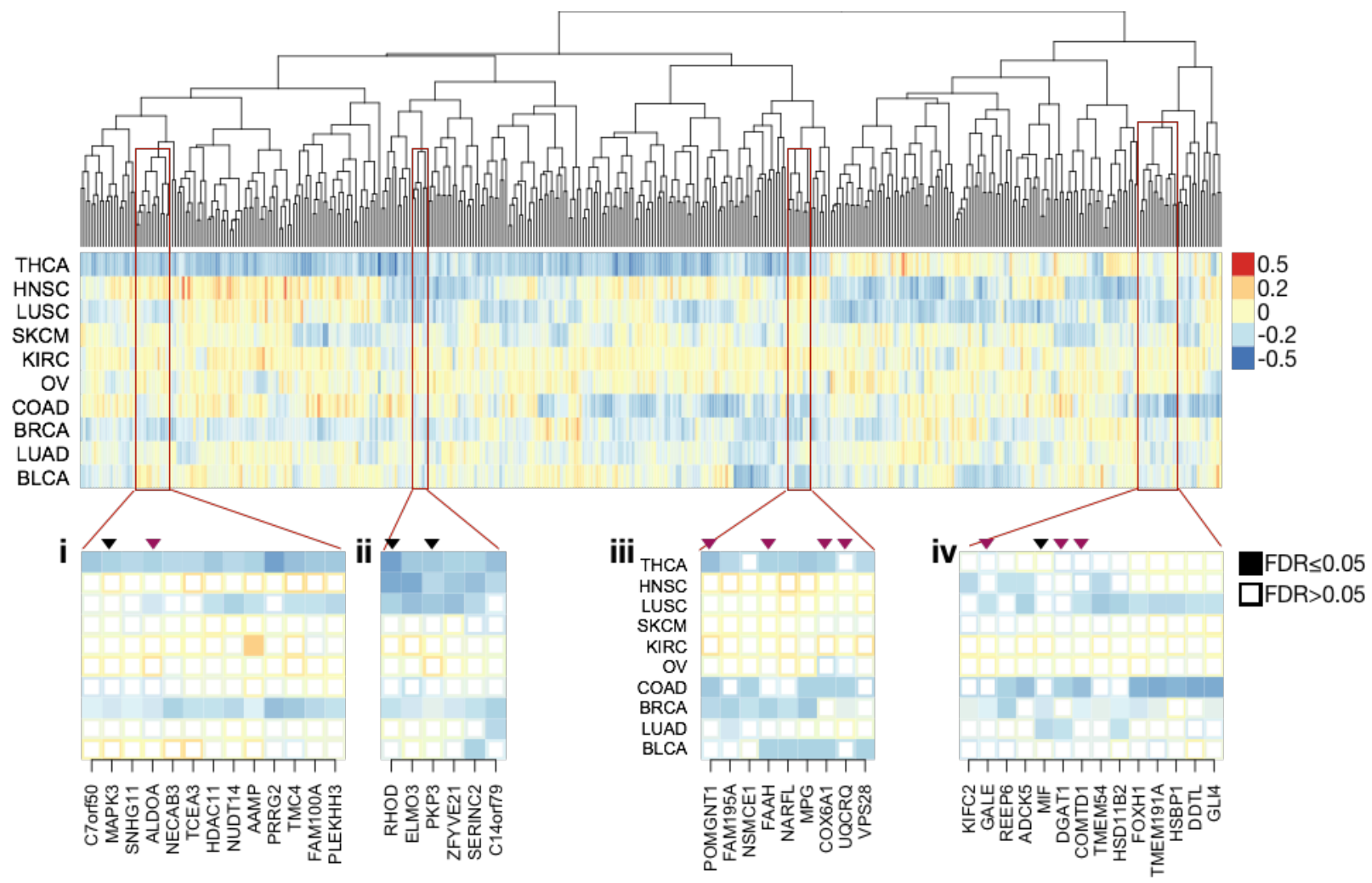

Supplementary Figure 4. Potential negative regulators for $\mathrm{T}$ cell activation in the tumor microenvironment. Genes with Spearman's correlation $\rho \leq-0.1$ and FDR $\leq 0.05$ in at least 3 cancer types were selected for visualization in the heatmap. Hierarchical clustering on $\rho$ was performed to order the genes into similar groups across different cancer types. Four representative clusters with putative oncogenes (labeled by black arrows) or recently identified metabolic enzymes (red arrows) were displayed as smaller heatmaps in the lower panels. Statistical significance was evaluated using partial Speaman's correlation test correcting for tumor purity, and FDR was performed using Benjamini-Hochberg procedure. 


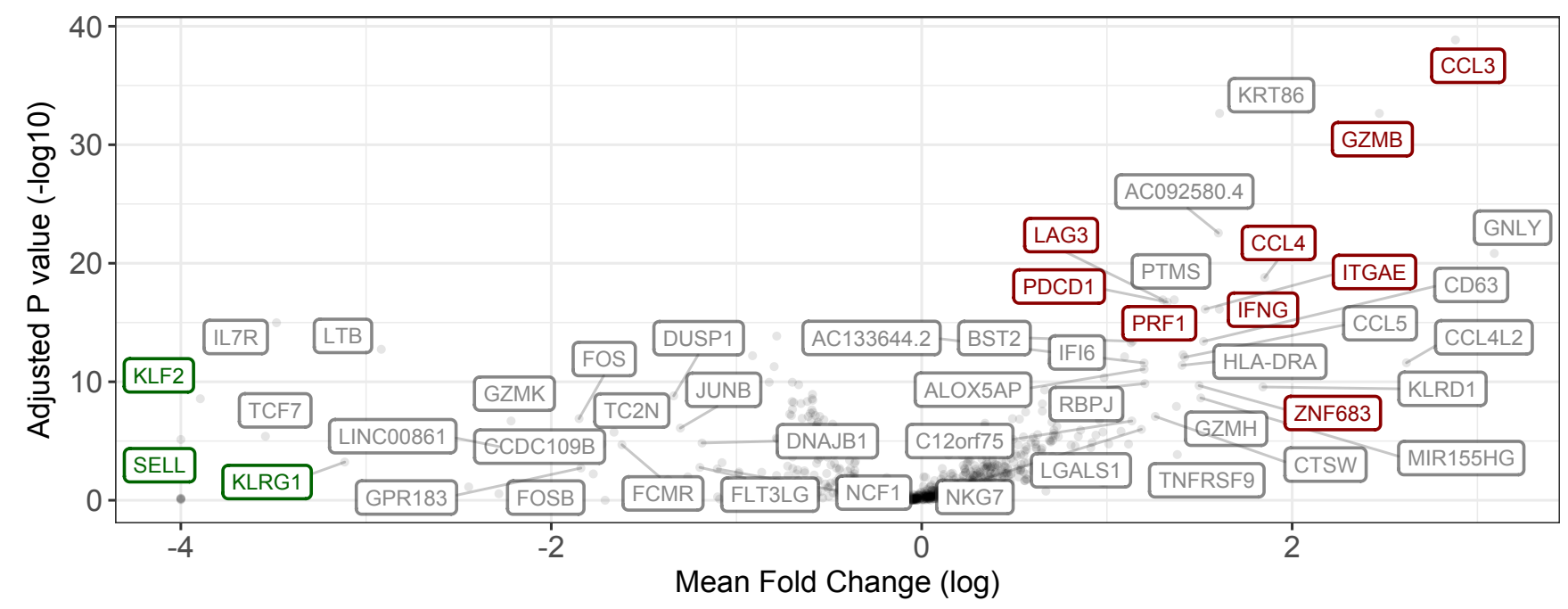

Supplementary Figure 5. Differentially expressed genes between our defined new group and other $\mathbf{T}$ cells. Wilcoxon rank sum test was applied to evaluate the statistical significance, and $\mathrm{p}$ values were corrected by Bejamini-Hochberg method. We labeled the genes with mean fold change greater than 3 or smaller than -3 , and FDR $<=0.01$. Established markers for tissue-resident memory $\mathrm{T}$ cells were highlighted with colors: red for up-regulation and green for down-regulation. 


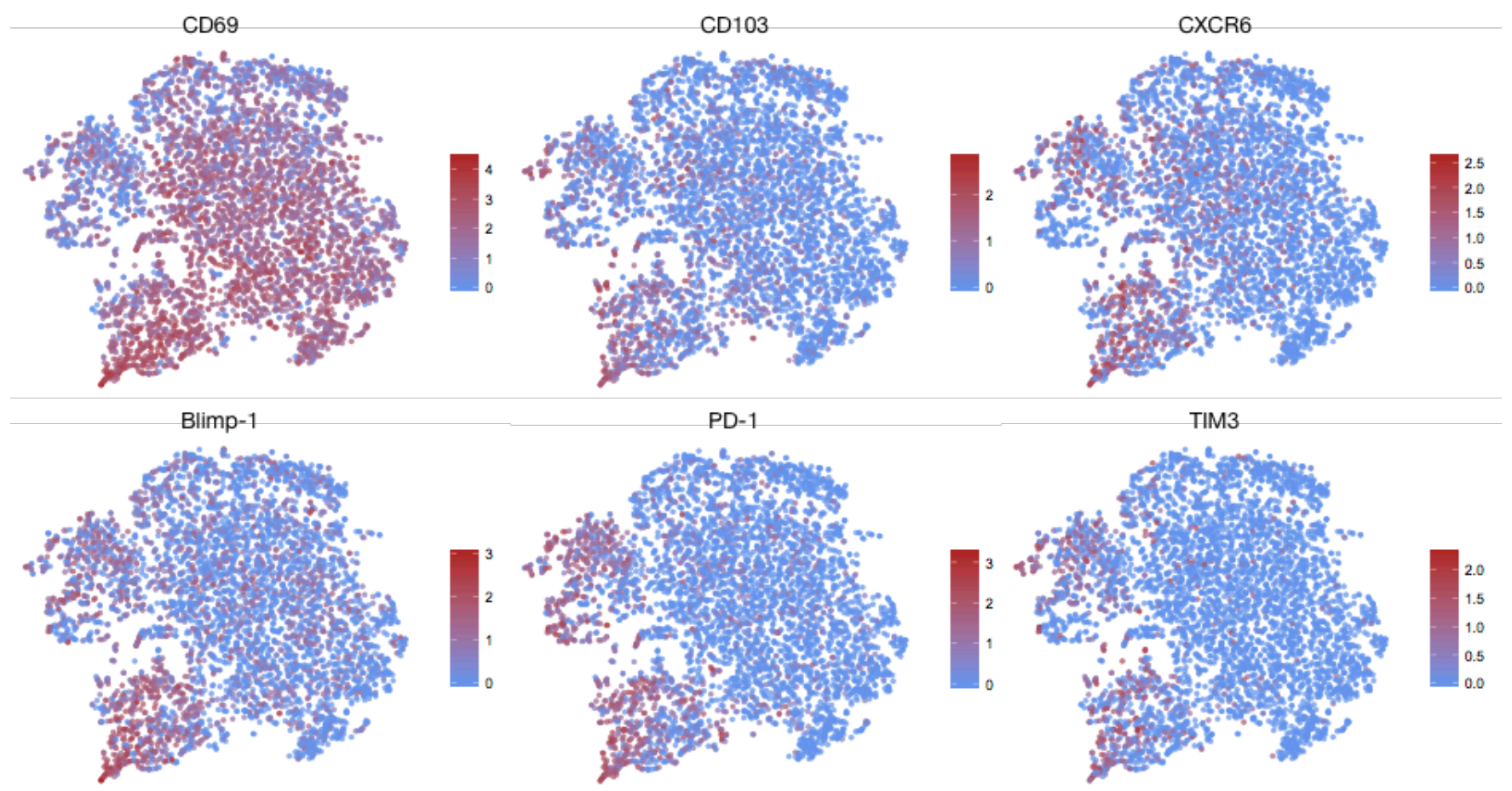

Supplementary Figure 6. Additional tSNE plot visualization for selected markers. Previously reported Trm markers including CD69 (general T cell activation and memory differentiation), CXCR6, Blimp-1 and CD103 (ITGAE) were visualized by tSNE plots. Expression patterns for two putative T cell exhaustion markers, PD-1 and TIM3 were also presented. Figure legend was in log scale. 

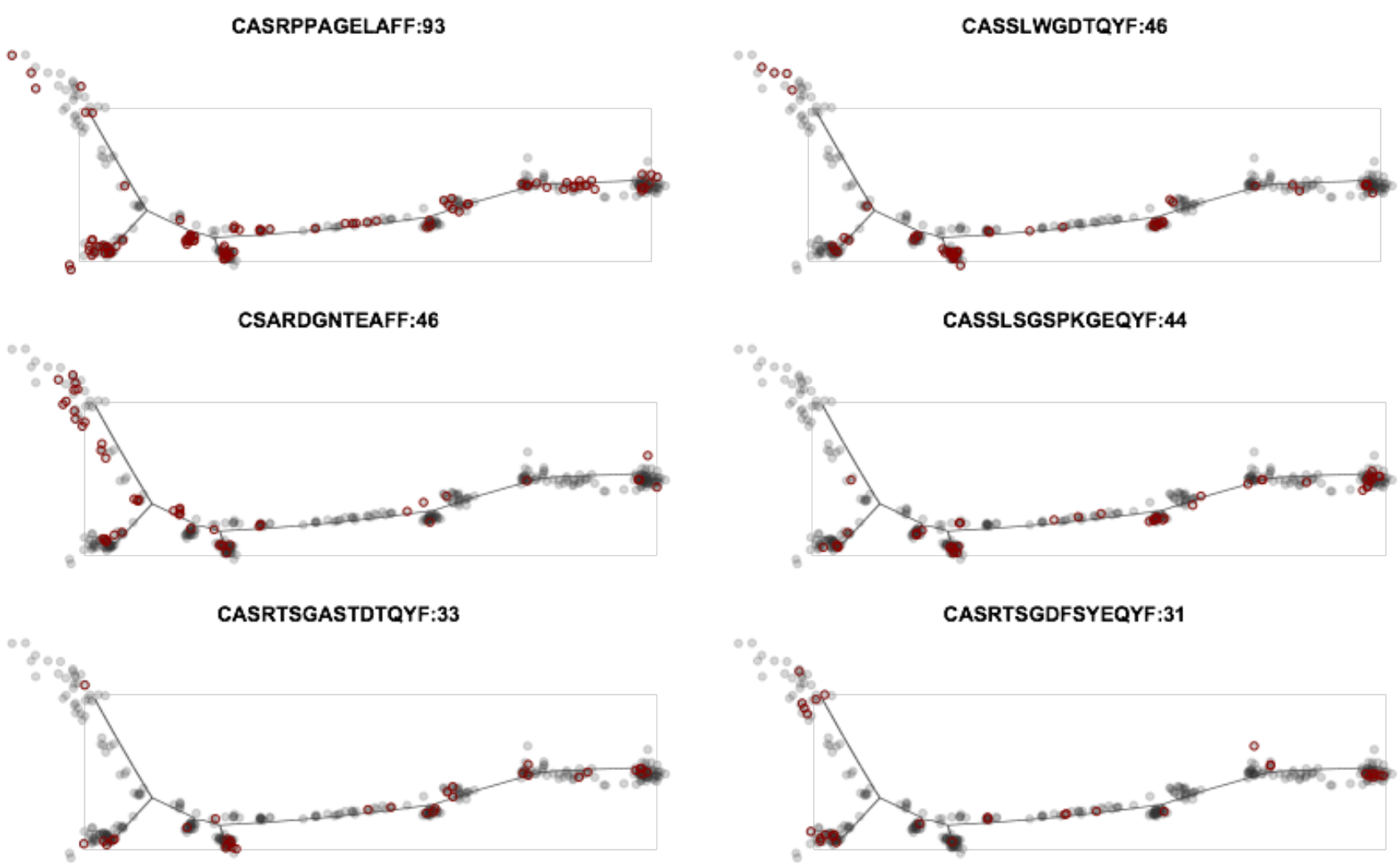

Supplementary Figure 7. Pseudotime trajectory plots for individual clonotypes in sample BC10. $\beta C D R 3$ sequences were displayed as figure titles and each point on the plot represent a cell. Red circles label cells with the corresponding CDR3 sequence. The numbers in the figure titles are the number of cells in the corresponding clonotype. Two evolutionary patterns were observed. Pattern 1 is from $\mathrm{T}_{\text {pre }}$ to $\mathrm{T}_{\mathrm{rm} 1}$, including clonotypes CASRPPAGELAFF, CASSLWGDTQYF, CASSLSGSPKGEQYF, CASRTSGASTDTQYF and CASRTSGDFSYEQYF. Pattern 2 is from $\mathrm{T}_{\mathrm{rm} 1}$ to $\mathrm{T}_{\mathrm{rm} 2 \text {, }}$ including clonotype CSARDGNTEAFF. 
a

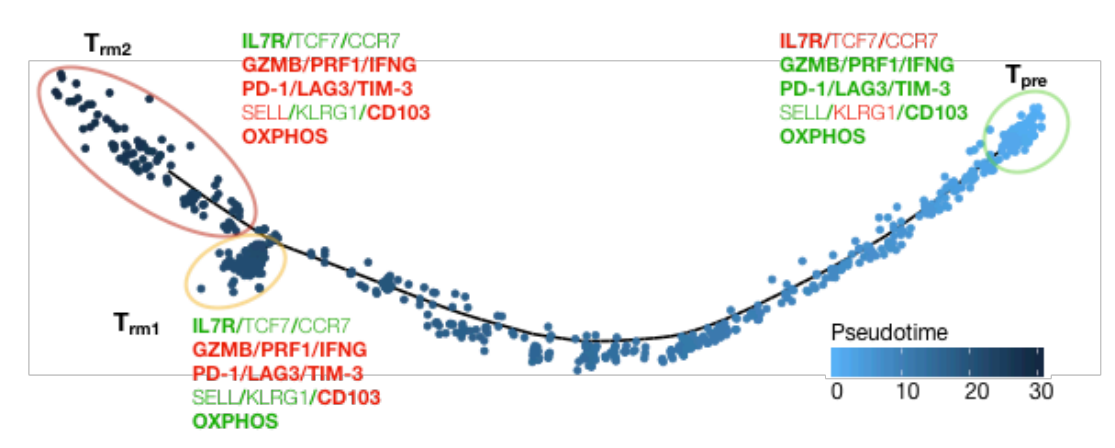

C

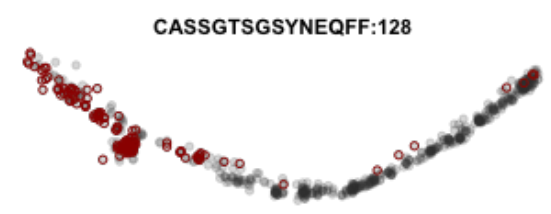

CASSLAPVSNYGYTF:37

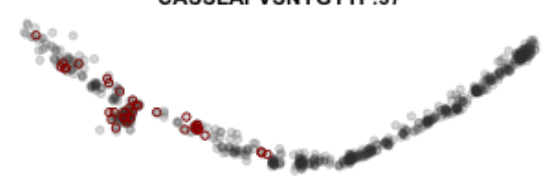

CQPVRDRGIYNEQFF:23

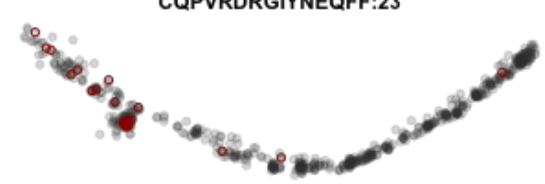

b

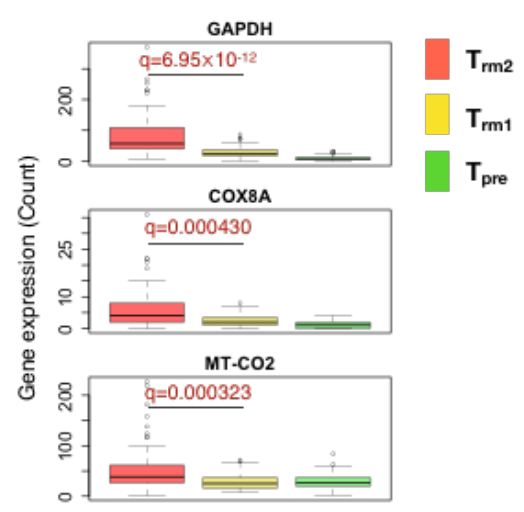

Supplementary Figure 8. Single cell trajectory analysis for breast cancer sample BC11. a) Same trajectory analysis in BC11, with exception that for the selected markers of the cell clusters, bold characters indicate FDR $<0.05$, where normal font otherwise. b) Boxplot for representative OXPHOS genes, with statistical significance evaluated by Wilcoxon rank sum test. c) Trajectory plots with individual clonotype overlaid by red circles. The number after each CDR3 sequence is the number of cells in the corresponding clonotype. Two evolutionary patterns were observed. Pattern 1 is from $T_{\text {pre }}$ to $T_{\mathrm{rm} 1}$, including clonotypes CASTDREGRYEQYF, CASSPDGKETQYF, CASSRDGQGNTIYF, CASSYSKVVLYGYTF and CASSPPSGSLGETQYF. Pattern 2 is from $\mathrm{T}_{\mathrm{rm} 1}$ to $\mathrm{T}_{\mathrm{rm} 2 \text {, }}$ including clonotypes CASSGTSGSYNEQFF, CASSLAPVSNYGYTF, CACSSGRYTGELFF and CQPVRDRGIYNEQFF. 
TCGA-BR-4256-01A-01R DYRK4 deletion at chr12:4719362

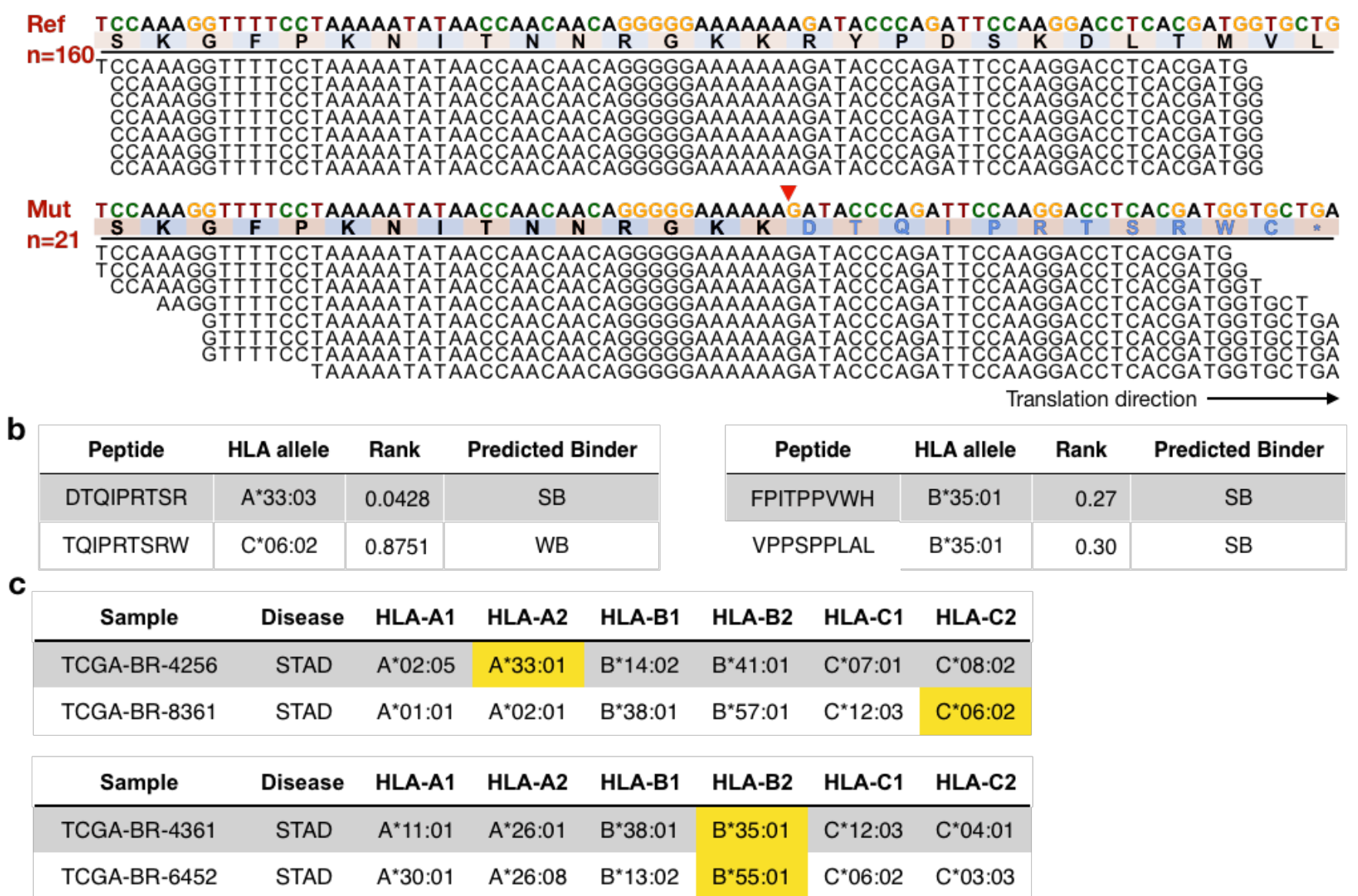

Supplementary Figure 9. Potential neoantigens generated from other top frameshift indels. a) Read pileup plot for gene DYRK4 frameshift deletion. The gene is forward translated. Site of deletion was labeled with red arrow. b-c) NetMHC predictions for binding peptides and TCGA sample HLA genotypes: left table in $\mathbf{b}$ and upper table in $\mathbf{c}$ for gene DYRK4; right table in $\mathbf{b}$ and lower table in c for gene RNF43 in Figure 3c. 
a

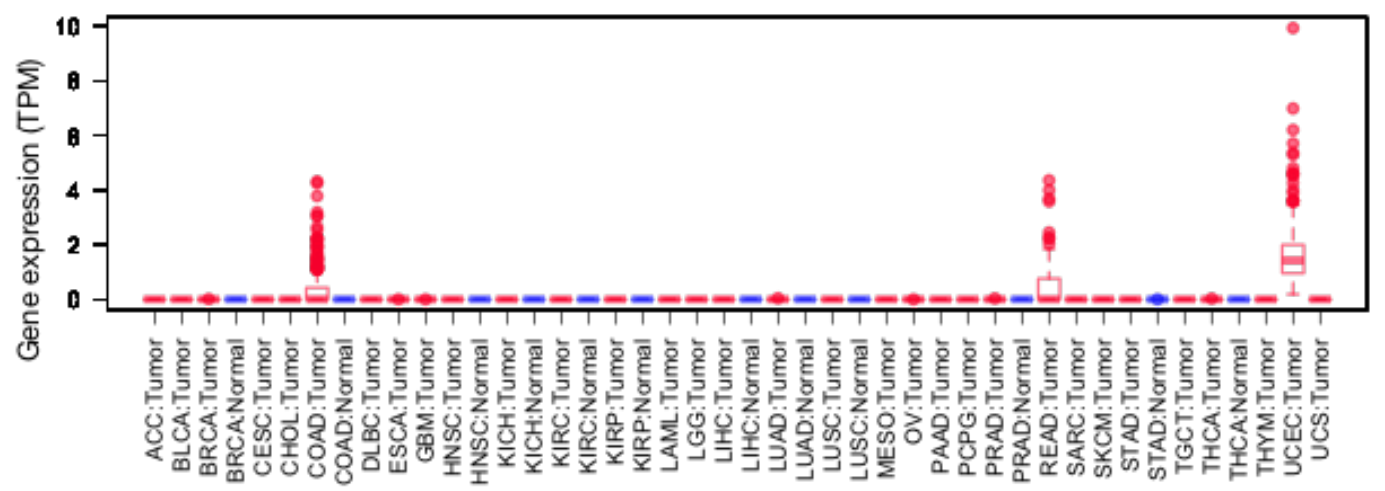

b

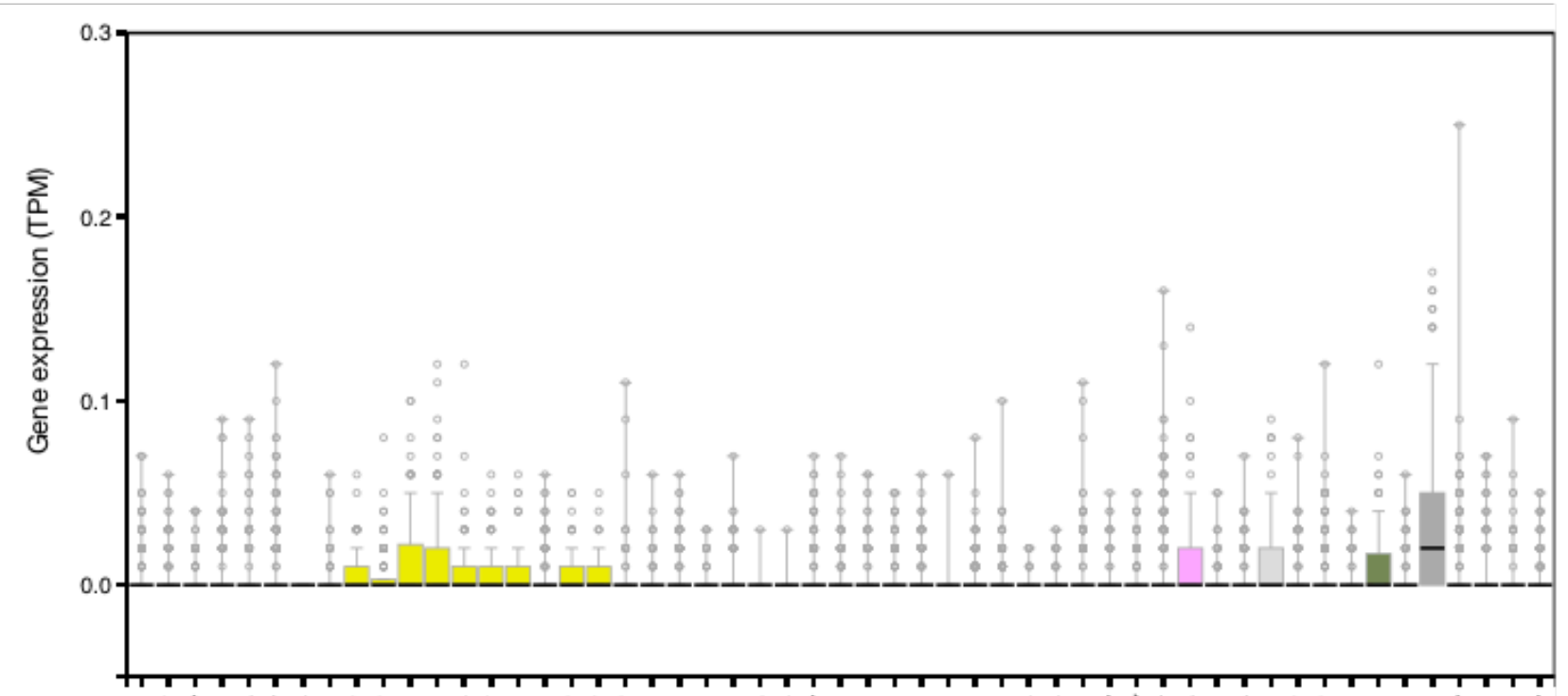

Supplementary Figure 10. HSFX1 expression across cancer types and normal tissues. a) TPM values for HSFX1 expression were displayed in boxplots across 32 cancer types. Adjacent normal samples with sufficient sample size $(\mathrm{n} \geq 20)$ were also included in the plot. b) TPM values for HSFX1 expression displayed in boxplot across 53 normal tissue types as reported by the GTEx data portal. Box colors distinguish major tissue types: yellow for brain, pink for ovary, light gray for prostate, dark gray for testis and olive green for spleen. 
a

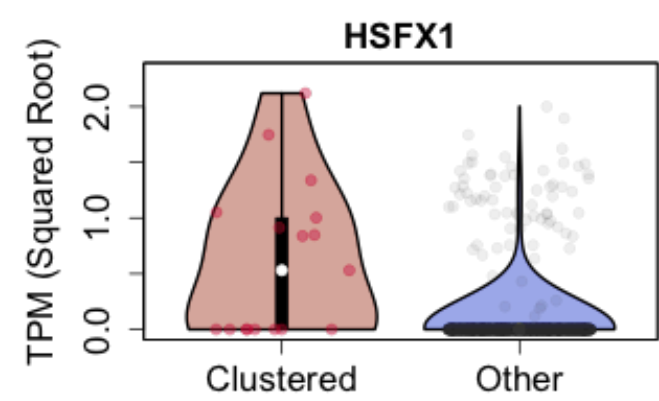

b

\begin{tabular}{|c|c|c|c|}
\hline \multicolumn{1}{|c|}{ Peptide } & HLA allele & Rank & Binder \\
\hline HSFX1: 384-392 & & & \\
\hline VMFPHLPAL & A $^{*} 02: 01$ & 0.02 & SB \\
\hline VMFPHLPAL & C $^{*} 07: 01$ & 0.25 & SB \\
\hline VMFPHLPAL & $C^{*} 07: 02$ & 0.125 & SB \\
\hline
\end{tabular}

C

\begin{tabular}{|c|c|c|c|c|c|c|c|c|}
\hline Sample & Disease & TPM & HLA-A1 & HLA-A2 & HLA-B1 & HLA-B2 & HLA-C1 & HLA-C2 \\
\hline TCGA & OAD & 0.28 & $A^{\star} 02: 01$ & $A^{\star} 25: 01$ & $B^{\star} 44: 02$ & ${ }^{\star} 51: 01$ & ${ }^{*} 07: 04$ & $C^{\star} 16: 02$ \\
\hline TCGA-AP-A1DM & UCEC & 1.00 & $A^{\star} 03: 01$ & $A^{*} 01: 01$ & $\mathrm{~B}^{\star} 08: 01$ & $B^{*} 35: 01$ & $C^{\star} 07: 01$ & $C^{\star} 04: 01$ \\
\hline TCGA-AA-A01K & COAD & 0.83 & $A^{*} 01: 01$ & $A^{*} 03: 01$ & $\mathrm{~B}^{\star} 07: 02$ & $B^{\star} 51: 01$ & $C^{\star} 07: 02$ & $C^{\star} 12: 02$ \\
\hline TCGA-AA-3684 & COAD & 1.10 & $A^{\star} 02: 01$ & $A^{\star} 24: 02$ & $B^{\star} 44: 05$ & $B^{\star} 52: 01$ & $C^{\star} 02: 02$ & $C^{\star} 12: 02$ \\
\hline TCGA-D1-A17F & UCEC & 1.79 & $A^{\star} 02: 01$ & $A^{\star} 02: 01$ & $B^{\star} 15: 01$ & $B^{\star} 51: 01$ & $C^{\star} 01: 02$ & $C^{\star} 03: 04$ \\
\hline TCGA-AA-3875 & COAD & 0.72 & $A^{\star} 01: 01$ & $A^{*} 68: 01$ & $\mathrm{~B}^{\star} 08: 01$ & $B^{*} 15: 01$ & $C^{\star} 03: 03$ & $C^{\star} 07: 01$ \\
\hline TCGA-AA-3848 & COAD & 3.05 & $A^{\star} 02: 01$ & $A^{\star} 29: 02$ & $B * 41: 02$ & $B * 44: 03$ & $C^{*} 16: 01$ & $C^{*} 17: 01$ \\
\hline TCGA-BG-A0MT & UCEC & 0.70 & $A^{\star} 02: 01$ & $A^{*} 02: 01$ & $B^{*} 44: 02$ & $B^{\star} 50: 01$ & $C^{\star} 05: 01$ & $C^{\star} 06: 02$ \\
\hline TCG & C & 4.49 & $A^{\star} 03: 01$ & $A^{\star} 24: 02$ & $\mathrm{~B}^{\star} 07: 02$ & 02 & 2 & 2 \\
\hline
\end{tabular}

\section{Supplementary Figure 11. Generation of a potentially immunogenic peptide by HSFX1}

expression. a) Violin plot showing significant difference in HSFX1 expression between clustered and non-clustered individuals from all TCGA cancers. 9 individuals with solved HLA genotypes have positive HSFX1 expression. b) NetMHC prediction for a HSFX1 derived 9-mer peptide, with strong binding affinities to 3 common HLA alleles. c) HLA genotype information table for the 9 individuals with HSFX1 expression shown in a). All 9 samples were colon or endometrial cancers, bearing at least one matched HLA binder(s) as shown in b) (highlighted in yellow). Expression levels in transcript per million (TPM) were also displayed in the table. 


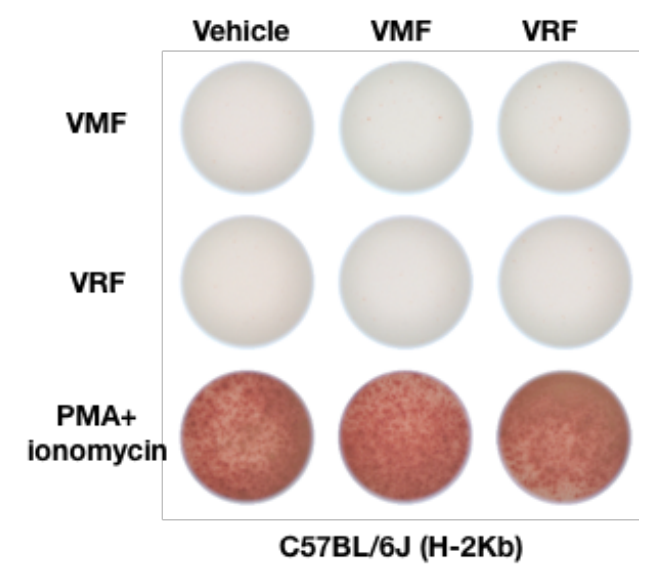

Supplementary Figure 12. Control experiment for HSFX1-derived peptide using naïve C57BL/6J mice. Same experiment as described in Figure 6a was performed using naïve immunocompetent C57BL/6 J mice ( $\mathrm{n}=4$, all female), and representative ELISPOT results were displayed. 
a

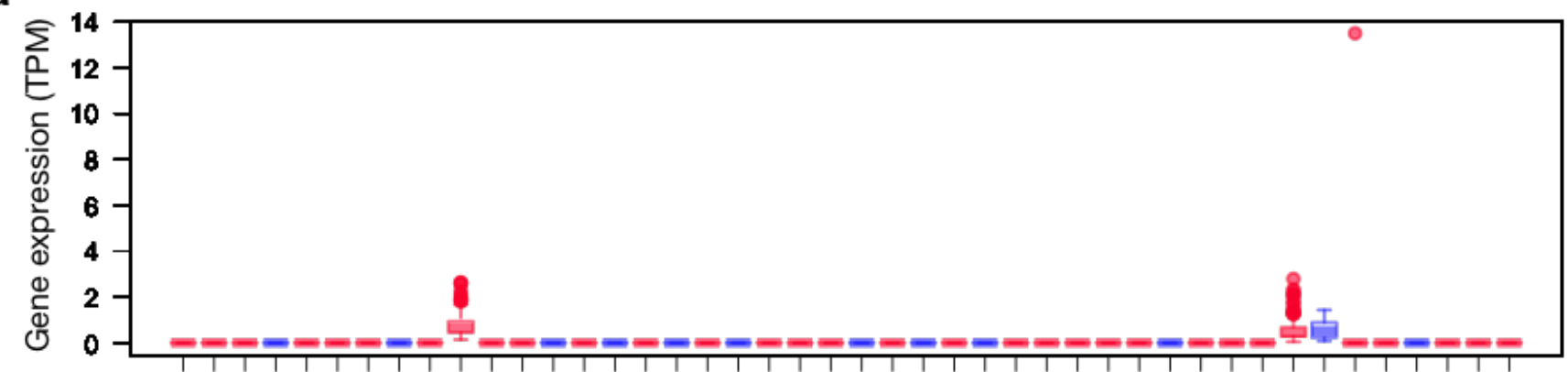

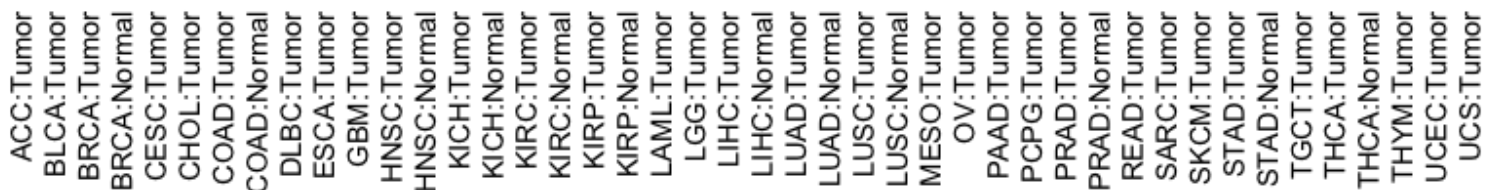

b

\begin{tabular}{|c|c|c|c|}
\hline Peptide & HLA allele & Rank & Predicted binder \\
\hline FETSDGRIY & B $^{\star} 44: 02$ & 0.6 & WB \\
\hline FETSDGRIY & B $^{\star} 44: 03$ & 0.4 & SB \\
\hline FETSDGRIY & C $^{*} 07: 02$ & 0.9 & WB \\
\hline
\end{tabular}

C

\begin{tabular}{|cccccccc|}
\hline Sample & Cancer & HLA-A1 & HLA-A2 & HLA-B1 & HLA-B2 & HLA-C1 & HLA-C2 \\
\hline TCGA-BR-8678 & STAD & $A^{\star} 11: 01$ & $A^{\star} 66: 01$ & $B^{\star} 55: 01$ & $B^{\star} 44: 02$ & $C^{\star} 07: 04$ & $C^{\star} 01: 02$ \\
\hline TCGA-BR-A4IZ & STAD & $A^{\star} 11: 01$ & $A^{\star} 33: 03$ & $B^{\star} 48: 01$ & $B^{\star} 44: 03$ & $C^{\star} 07: 06$ & $C^{\star} 08: 01$ \\
\hline TCGA-D7-8572 & STAD & $A^{\star} 68: 01$ & $A^{\star} 29: 02$ & $B^{\star} 27: 02$ & $B^{\star} 44: 03$ & $C^{\star} 16: 01$ & $C^{\star} 02: 02$ \\
\hline TCGA-BR-4267 & STAD & $A^{\star} 02: 01$ & $A^{\star} 02: 01$ & $B^{\star} 07: 02$ & $B^{\star} 07: 02$ & $C^{\star} 07: 02$ & $C^{\star} 07: 02$ \\
TCGA-BR-8484 & STAD & $A^{\star} 03: 01$ & $A^{\star} 02: 01$ & $B^{\star} 07: 02$ & $B^{\star} 40: 01$ & $C^{\star} 07: 02$ & $C^{\star} 03: 04$ \\
\hline
\end{tabular}

Supplementary Figure 13. Identification of TSSK2 as a potential cancer-associated antigen. a) Boxplot for TSSK2 expression across different tumor and normal tissues. b-c) NetMHC predicted binding peptide information and HLA genotypes for samples with TSSK2 expression. 
a

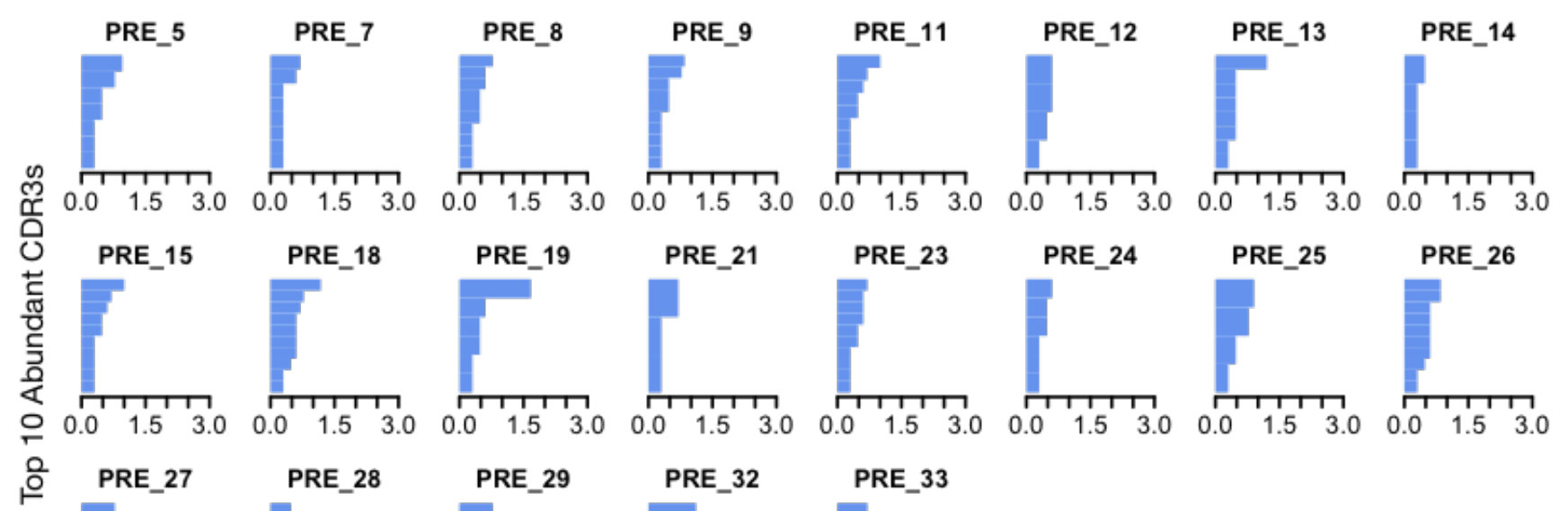

b
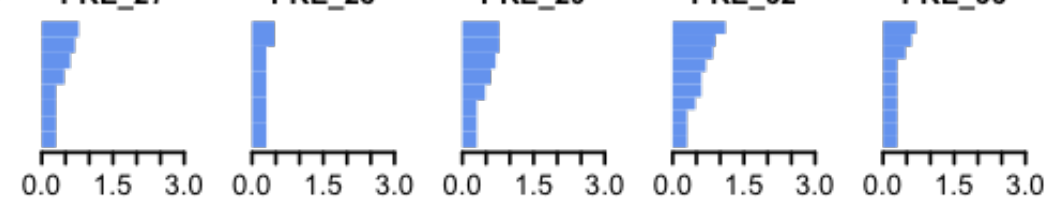

Read Count $(\log 10)$

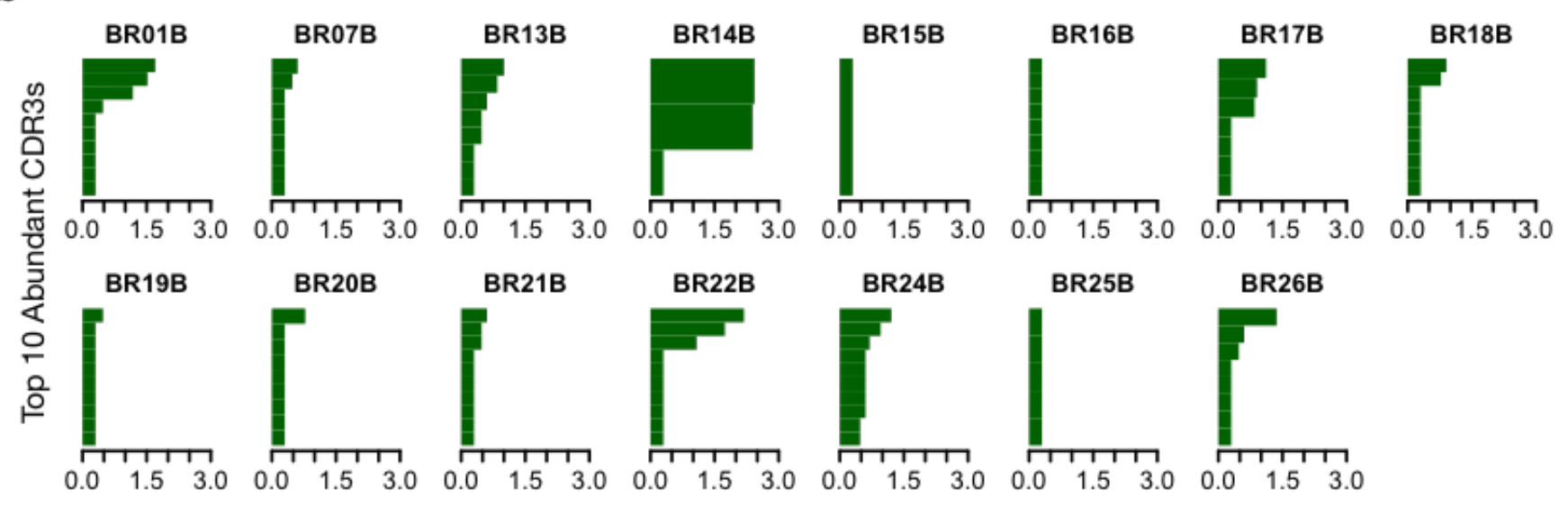

Read Count $(\log 10)$

Supplementary Figure 14. Cancer-associated $\mathrm{T}$ cell clonal frequencies in early and late stage tumors. a-b) Barplots showing the read counts for top 10 cancer-associated CDR3s in samples of the melanoma (a) or early breast cancer (b) cohorts. Since the library sizes for TCR-seq data in the melanoma cohort were bigger than those for the early breast cancer cohort, to ensure fair comparison, we downsampled the libraries of the melanoma samples to $N$, which randomly sampled from Uniform distribution $(34383,156524)$. The upper and lower limits mark the range of library sizes for early breast cancer samples. Specifically, for each library, we randomly sampled an integer ( $\mathrm{N}$ ) from that range, and downsampled the initial library to $\mathrm{N}$ reads, and estimated CDR3 frequencies (Methods). The above range matches the library sizes for the early breast cancer cohort. 
Supplementary Table 1. Information for the 15 selected antigens for methodology performance evaluation.

Supplementary Table 2. Summary of association analysis between gene expression levels and clustered CDR3 count in each individual.

Supplementary Table 3. Summary of differential gene expression analysis between the single cells from new defined group and others, with fold change and FDR estimations.

Supplementary Table 4. HLA genotype information for patients carrying correlated pairs of predicted neoantigens and clustered CDR3s.

Supplementary Table 5. Summary of differential gene expression analysis between each CDR3 cluster and other TCGA tumors, with fold change and FDR estimations.

Supplementary Dataset 1. TCR clusters obtained from the top 5000 most abundant clonotypes of 666 HCMV cohort. (Due to file size limit, in this submission we only included 3 files for review. Format of the remaining files is the same.)

Supplementary Dataset 2. Public non-specific TCRs profiled using random triplets with unmatched HLA alleles from the HCMV cohort. 


\section{Reference}

1. $\quad$ Ahmadzadeh, M., Johnson, L.A., Heemskerk, B., Wunderlich, J.R., Dudley, M.E., White, D.E. \& Rosenberg, S.A. Tumor antigen-specific CD8 $\mathrm{T}$ cells infiltrating the tumor express high levels of PD-1 and are functionally impaired. Blood 114, 1537-44 (2009).10.1182/blood-2008-12-195792. 2927090.

2. Coulie, P.G., Van den Eynde, B.J., van der Bruggen, P. \& Boon, T. Tumour antigens recognized by Tymphocytes: at the core of cancer immunotherapy. Nat Rev Cancer 14,135-46 (2014).10.1038/nrc3670.

3. Gooden, M.J., de Bock, G.H., Leffers, N., Daemen, T. \& Nijman, H.W. The prognostic influence of tumourinfiltrating lymphocytes in cancer: a systematic review with meta-analysis. Br J Cancer 105, 93-103 (2011).10.1038/bjc.2011.189. 3137407.

4. Gubin, M.M., Zhang, X., Schuster, H., Caron, E., Ward, J.P., Noguchi, T., Ivanova, Y., Hundal, J., Arthur, C.D., Krebber, W.J., Mulder, G.E., Toebes, M., Vesely, M.D., Lam, S.S., Korman, A.J., Allison, J.P., Freeman, G.J., Sharpe, A.H., Pearce, E.L., Schumacher, T.N., Aebersold, R., Rammensee, H.G., Melief, C.J., Mardis, E.R., Gillanders, W.E., Artyomov, M.N. \& Schreiber, R.D. Checkpoint blockade cancer immunotherapy targets tumour-specific mutant antigens. Nature 515, 577-81 (2014).10.1038/nature13988. 4279952.

5. Pardoll, D.M. The blockade of immune checkpoints in cancer immunotherapy. Nat Rev Cancer 12, 252-64 (2012).10.1038/nrc3239. 4856023.

6. Tang, H., Wang, Y., Chlewicki, L.K., Zhang, Y., Guo, J., Liang, W., Wang, J., Wang, X. \& Fu, Y.X. Facilitating T Cell Infiltration in Tumor Microenvironment Overcomes Resistance to PD-L1 Blockade. Cancer Cell 30, 500 (2016).10.1016/j.ccell.2016.08.011.

7. Kalos, M. \& June, C.H. Adoptive T cell transfer for cancer immunotherapy in the era of synthetic biology. Immunity 39, 49-60 (2013).10.1016/j.immuni.2013.07.002.3809038.

8. Rosenberg, S.A., Restifo, N.P., Yang, J.C., Morgan, R.A. \& Dudley, M.E. Adoptive cell transfer: a clinical path to effective cancer immunotherapy. Nat Rev Cancer 8, 299-308 (2008).10.1038/nrc2355. 2553205.

9. Stronen, E., Toebes, M., Kelderman, S., van Buuren, M.M., Yang, W., van Rooij, N., Donia, M., Boschen, M.L., Lund-Johansen, F., Olweus, J. \& Schumacher, T.N. Targeting of cancer neoantigens with donor-derived T cell receptor repertoires. Science 352, 1337-41 (2016).10.1126/science.aaf2288.

10. Tran, E., Turcotte, S., Gros, A., Robbins, P.F., Lu, Y.C., Dudley, M.E., Wunderlich, J.R., Somerville, R.P., Hogan, K., Hinrichs, C.S., Parkhurst, M.R., Yang, J.C. \& Rosenberg, S.A. Cancer immunotherapy based on mutation-specific CD4+ T cells in a patient with epithelial cancer. Science 344, 641-5 (2014).10.1126/science.1251102.

11. Greenman, C., Stephens, P., Smith, R., Dalgliesh, G.L., Hunter, C., Bignell, G., Davies, H., Teague, J., Butler, A., Stevens, C., Edkins, S., O'Meara, S., Vastrik, I., Schmidt, E.E., Avis, T., Barthorpe, S., Bhamra, G., Buck, G., Choudhury, B., Clements, J., Cole, J., Dicks, E., Forbes, S., Gray, K., Halliday, K., Harrison, R., Hills, K., Hinton, J., Jenkinson, A., Jones, D., Menzies, A., Mironenko, T., Perry, J., Raine, K., Richardson, D., Shepherd, R., Small, A., Tofts, C., Varian, J., Webb, T., West, S., Widaa, S., Yates, A., Cahill, D.P., Louis, D.N., Goldstraw, P., Nicholson, A.G., Brasseur, F., Looijenga, L., Weber, B.L., Chiew, Y.E., DeFazio, A., Greaves, M.F., Green, A.R., Campbell, P., Birney, E., Easton, D.F., Chenevix-Trench, G., Tan, M.H., Khoo, S.K., Teh, B.T., Yuen, S.T., Leung, S.Y., Wooster, R., Futreal, P.A. \& Stratton, M.R. Patterns of somatic mutation in human cancer genomes. Nature 446, 153-8 (2007).10.1038/nature05610. 2712719.

12. Pleasance, E.D., Cheetham, R.K., Stephens, P.J., McBride, D.J., Humphray, S.J., Greenman, C.D., Varela, I., Lin, M.L., Ordonez, G.R., Bignell, G.R., Ye, K., Alipaz, J., Bauer, M.J., Beare, D., Butler, A., Carter, R.J., Chen, L., Cox, A.J., Edkins, S., Kokko-Gonzales, P.I., Gormley, N.A., Grocock, R.J., Haudenschild, C.D., Hims, M.M., James, T., Jia, M., Kingsbury, Z., Leroy, C., Marshall, J., Menzies, A., Mudie, L.J., Ning, Z., Royce, T., Schulz-Trieglaff, O.B., Spiridou, A., Stebbings, L.A., Szajkowski, L., Teague, J., Williamson, D., Chin, L., Ross, M.T., Campbell, P.J., Bentley, D.R., Futreal, P.A. \& Stratton, M.R. A comprehensive catalogue of somatic mutations from a human cancer genome. Nature 463, 191-6 (2010).10.1038/nature08658. 3145108.

13. Saeterdal, I., Bjorheim, J., Lislerud, K., Gjertsen, M.K., Bukholm, I.K., Olsen, O.C., Nesland, J.M., Eriksen, J.A., Moller, M., Lindblom, A. \& Gaudernack, G. Frameshift-mutation-derived peptides as tumor-specific antigens in inherited and spontaneous colorectal cancer. Proc Natl Acad Sci U S A 98, 13255-60 (2001).10.1073/pnas.231326898. 60857.

14. Turajlic, S., Litchfield, K., Xu, H., Rosenthal, R., McGranahan, N., Reading, J.L., Wong, Y.N.S., Rowan, A., Kanu, N., Al Bakir, M., Chambers, T., Salgado, R., Savas, P., Loi, S., Birkbak, N.J., Sansregret, L., Gore, M., Larkin, J., Quezada, S.A. \& Swanton, C. Insertion-and-deletion-derived tumour-specific neoantigens and the immunogenic phenotype: a pan-cancer analysis. Lancet Oncol 18, 1009-1021 (2017).10.1016/S1470-2045(17)30516-8.

15. Scanlan, M.J., Gure, A.O., Jungbluth, A.A., Old, L.J. \& Chen, Y.T. Cancer/testis antigens: an expanding family of targets for cancer immunotherapy. Immunol Rev 188, 22-32 (2002).

16. Simpson, A.J., Caballero, O.L., Jungbluth, A., Chen, Y.T. \& Old, L.J. Cancer/testis antigens, gametogenesis and cancer. Nat Rev Cancer 5, 615-25 (2005).10.1038/nrc1669. 
17. Doyle, H.A. \& Mamula, M.J. Post-translational protein modifications in antigen recognition and autoimmunity. Trends Immunol 22, 443-9 (2001).

18. Dvinge, H. \& Bradley, R.K. Widespread intron retention diversifies most cancer transcriptomes. Genome Med 7, 45 (2015).10.1186/s13073-015-0168-9. 4480902.

19. Sahu, A., Singhal, U. \& Chinnaiyan, A.M. Long noncoding RNAs in cancer: from function to translation. Trends Cancer 1, 93-109 (2015).10.1016/j.trecan.2015.08.010. 4672369.

20. Cascio, S., Zhang, L. \& Finn, O.J. MUC1 protein expression in tumor cells regulates transcription of proinflammatory cytokines by forming a complex with nuclear factor-kappaB p65 and binding to cytokine promoters: importance of extracellular domain. J Biol Chem 286, 42248-56 (2011).10.1074/jbc.M111.297630. 3234962.

21. Arstila, T.P., Casrouge, A., Baron, V., Even, J., Kanellopoulos, J. \& Kourilsky, P. A direct estimate of the human alphabeta T cell receptor diversity. Science 286, 958-61 (1999).

22. Glanville, J., Huang, H., Nau, A., Hatton, O., Wagar, L.E., Rubelt, F., Ji, X., Han, A., Krams, S.M., Pettus, C., Haas, N., Arlehamn, C.S.L., Sette, A., Boyd, S.D., Scriba, T.J., Martinez, O.M. \& Davis, M.M. Identifying specificity groups in the T cell receptor repertoire. Nature 547, 94-98 (2017).10.1038/nature22976. 5794212.

23. Dash, P., Fiore-Gartland, A.J., Hertz, T., Wang, G.C., Sharma, S., Souquette, A., Crawford, J.C., Clemens, E.B., Nguyen, T.H.O., Kedzierska, K., La Gruta, N.L., Bradley, P. \& Thomas, P.G. Quantifiable predictive features define epitope-specific T cell receptor repertoires. Nature 547, 89-93 (2017).10.1038/nature22383. 5616171.

24. Beausang, J.F., Wheeler, A.J., Chan, N.H., Hanft, V.R., Dirbas, F.M., Jeffrey, S.S. \& Quake, S.R. T cell receptor sequencing of early-stage breast cancer tumors identifies altered clonal structure of the T cell repertoire. Proc Natl Acad Sci U S A 114, E10409-E10417 (2017).10.1073/pnas.1713863114. 5715779.

25. Robert, L., Tsoi, J., Wang, X., Emerson, R., Homet, B., Chodon, T., Mok, S., Huang, R.R., Cochran, A.J., CominAnduix, B., Koya, R.C., Graeber, T.G., Robins, H. \& Ribas, A. CTLA4 blockade broadens the peripheral T-cell receptor repertoire. Clin Cancer Res 20, 2424-32 (2014).10.1158/1078-0432.CCR-13-2648. 4008652.

26. Li, B., Li, T., Pignon, J.C., Wang, B., Wang, J., Shukla, S.A., Dou, R., Chen, Q., Hodi, F.S., Choueiri, T.K., Wu, C., Hacohen, N., Signoretti, S., Liu, J.S. \& Liu, X.S. Landscape of tumor-infiltrating T cell repertoire of human cancers. Nat Genet 48, 725-32 (2016).10.1038/ng.3581. 5298896.

27. Li, B., Li, T., Wang, B., Dou, R., Zhang, J., Liu, J.S. \& Liu, X.S. Ultrasensitive detection of TCR hypervariable-region sequences in solid-tissue RNA-seq data. Nat Genet 49, 482-483 (2017).10.1038/ng.3820.

28. Lefranc, M.P., Giudicelli, V., Duroux, P., Jabado-Michaloud, J., Folch, G., Aouinti, S., Carillon, E., Duvergey, H., Houles, A., Paysan-Lafosse, T., Hadi-Saljoqi, S., Sasorith, S., Lefranc, G. \& Kossida, S. IMGT(R), the international ImMunoGeneTics information system(R) 25 years on. Nucleic Acids Res 43, D413-22 (2015).10.1093/nar/gku1056. 4383898.

29. Madi, A., Shifrut, E., Reich-Zeliger, S., Gal, H., Best, K., Ndifon, W., Chain, B., Cohen, I.R. \& Friedman, N. T-cell receptor repertoires share a restricted set of public and abundant CDR3 sequences that are associated with self-related immunity. Genome Res 24, 1603-12 (2014).10.1101/gr.170753.113. 4199372.

30. Emerson, R.O., DeWitt, W.S., Vignali, M., Gravley, J., Hu, J.K., Osborne, E.J., Desmarais, C., Klinger, M., Carlson, C.S., Hansen, J.A., Rieder, M. \& Robins, H.S. Immunosequencing identifies signatures of cytomegalovirus exposure history and HLA-mediated effects on the T cell repertoire. Nat Genet 49, 659-665 (2017).10.1038/ng.3822.

31. Adams, J.J., Narayanan, S., Liu, B., Birnbaum, M.E., Kruse, A.C., Bowerman, N.A., Chen, W., Levin, A.M., Connolly, J.M., Zhu, C., Kranz, D.M. \& Garcia, K.C. T cell receptor signaling is limited by docking geometry to peptidemajor histocompatibility complex. Immunity 35, 681-93 (2011).10.1016/j.immuni.2011.09.013. 3253265.

32. Birnbaum, M.E., Mendoza, J.L., Sethi, D.K., Dong, S., Glanville, J., Dobbins, J., Ozkan, E., Davis, M.M., Wucherpfennig, K.W. \& Garcia, K.C. Deconstructing the peptide-MHC specificity of T cell recognition. Cell 157, 1073-87 (2014).10.1016/j.cell.2014.03.047. 4071348.

33. Gee, M.H., Han, A., Lofgren, S.M., Beausang, J.F., Mendoza, J.L., Birnbaum, M.E., Bethune, M.T., Fischer, S., Yang, X., Gomez-Eerland, R., Bingham, D.B., Sibener, L.V., Fernandes, R.A., Velasco, A., Baltimore, D., Schumacher, T.N., Khatri, P., Quake, S.R., Davis, M.M. \& Garcia, K.C. Antigen Identification for Orphan T Cell Receptors Expressed on Tumor-Infiltrating Lymphocytes. Cell 172, 549-563 e16 (2018).10.1016/j.cell.2017.11.043. 5786495.

34. Shugay, M., Bagaev, D.V., Zvyagin, I.V., Vroomans, R.M., Crawford, J.C., Dolton, G., Komech, E.A., Sycheva, A.L., Koneva, A.E., Egorov, E.S., Eliseev, A.V., Van Dyk, E., Dash, P., Attaf, M., Rius, C., Ladell, K., McLaren, J.E., Matthews, K.K., Clemens, E.B., Douek, D.C., Luciani, F., van Baarle, D., Kedzierska, K., Kesmir, C., Thomas, P.G., Price, D.A., Sewell, A.K. \& Chudakov, D.M. VDJdb: a curated database of T-cell receptor sequences with known antigen specificity. Nucleic Acids Res 46, D419-D427 (2018).10.1093/nar/gkx760. 5753233.

35. Li, B., Severson, E., Pignon, J.C., Zhao, H., Li, T., Novak, J., Jiang, P., Shen, H., Aster, J.C., Rodig, S., Signoretti, S., Liu, J.S. \& Liu, X.S. Comprehensive analyses of tumor immunity: implications for cancer immunotherapy. Genome Biol 17, 174 (2016).10.1186/s13059-016-1028-7. 4993001. 
36. Subramanian, A., Tamayo, P., Mootha, V.K., Mukherjee, S., Ebert, B.L., Gillette, M.A., Paulovich, A., Pomeroy, S.L., Golub, T.R., Lander, E.S. \& Mesirov, J.P. Gene set enrichment analysis: a knowledge-based approach for interpreting genome-wide expression profiles. Proc Natl Acad Sci U S A 102, 15545-50 (2005).10.1073/pnas.0506580102.1239896.

37. Barnes, M.J., Li, C.M., Xu, Y., An, J., Huang, Y. \& Cyster, J.G. The lysophosphatidylserine receptor GPR174 constrains regulatory T cell development and function. J Exp Med 212,1011-20 (2015).10.1084/jem.20141827. 4493414.

38. Burotto, M., Chiou, V.L., Lee, J.M. \& Kohn, E.C. The MAPK pathway across different malignancies: a new perspective. Cancer 120, 3446-56 (2014).10.1002/cncr.28864. 4221543.

39. Ebert, P.J.R., Cheung, J., Yang, Y., McNamara, E., Hong, R., Moskalenko, M., Gould, S.E., Maecker, H., Irving, B.A., Kim, J.M., Belvin, M. \& Mellman, I. MAP Kinase Inhibition Promotes T Cell and Anti-tumor Activity in Combination with PD-L1 Checkpoint Blockade. Immunity 44, 609-621 (2016).10.1016/j.immuni.2016.01.024.

40. Cascone, T., McKenzie, J.A., Mbofung, R.M., Punt, S., Wang, Z., Xu, C., Williams, L.J., Wang, Z., Bristow, C.A., Carugo, A., Peoples, M.D., Li, L., Karpinets, T., Huang, L., Malu, S., Creasy, C., Leahey, S.E., Chen, J., Chen, Y., Pelicano, H., Bernatchez, C., Gopal, Y.N.V., Heffernan, T.P., Hu, J., Wang, J., Amaria, R.N., Garraway, L.A., Huang, P., Yang, P., Wistuba, II, Woodman, S.E., Roszik, J., Davis, R.E., Davies, M.A., Heymach, J.V., Hwu, P. \& Peng, W. Increased Tumor Glycolysis Characterizes Immune Resistance to Adoptive T Cell Therapy. Cell Metab 27, $977-$ 987 e4 (2018).10.1016/j.cmet.2018.02.024. 5932208.

41. Chaker, M., Minden, A., Chen, S., Weiss, R.H., Chini, E.N., Mahipal, A. \& Azmi, A.S. Rho GTPase effectors and NAD metabolism in cancer immune suppression. Expert Opin Ther Targets 22, 9-17 (2018).10.1080/14728222.2018.1413091.

42. Renner, K., Singer, K., Koehl, G.E., Geissler, E.K., Peter, K., Siska, P.J. \& Kreutz, M. Metabolic Hallmarks of Tumor and Immune Cells in the Tumor Microenvironment. Front Immunol 8, 248 (2017).10.3389/fimmu.2017.00248.5340776.

43. Azizi, E., Carr, A.J., Plitas, G., Cornish, A.E., Konopacki, C., Prabhakaran, S., Nainys, J., Wu, K., Kiseliovas, V., Setty, M., Choi, K., Fromme, R.M., Dao, P., McKenney, P.T., Wasti, R.C., Kadaveru, K., Mazutis, L., Rudensky, A.Y. \& Pe'er, D. Single-Cell Map of Diverse Immune Phenotypes in the Breast Tumor Microenvironment. Cell (2018).10.1016/j.cell.2018.05.060.

44. van der Maaten, L.J.P. \& Hinton, G.E. Visualizing High-Dimensional Data Using t-SNE. Journal of Machine Learning Research 9, 2579-2605 (2008).

45. Savas, P., Virassamy, B., Ye, C., Salim, A., Mintoff, C.P., Caramia, F., Salgado, R., Byrne, D.J., Teo, Z.L., Dushyanthen, S., Byrne, A., Wein, L., Luen, S.J., Poliness, C., Nightingale, S.S., Skandarajah, A.S., Gyorki, D.E., Thornton, C.M., Beavis, P.A., Fox, S.B., Kathleen Cuningham Foundation Consortium for Research into Familial Breast, C., Darcy, P.K., Speed, T.P., Mackay, L.K., Neeson, P.J. \& Loi, S. Single-cell profiling of breast cancer T cells reveals a tissue-resident memory subset associated with improved prognosis. Nat Med 24, 986-993 (2018).10.1038/s41591-018-0078-7.

46. Kumar, B.V., Ma, W., Miron, M., Granot, T., Guyer, R.S., Carpenter, D.J., Senda, T., Sun, X., Ho, S.H., Lerner, H., Friedman, A.L., Shen, Y. \& Farber, D.L. Human Tissue-Resident Memory T Cells Are Defined by Core Transcriptional and Functional Signatures in Lymphoid and Mucosal Sites. Cell Rep 20, 2921-2934 (2017).10.1016/j.celrep.2017.08.078. 5646692.

47. Mackay, L.K., Minnich, M., Kragten, N.A., Liao, Y., Nota, B., Seillet, C., Zaid, A., Man, K., Preston, S., Freestone, D., Braun, A., Wynne-Jones, E., Behr, F.M., Stark, R., Pellicci, D.G., Godfrey, D.I., Belz, G.T., Pellegrini, M., Gebhardt, T., Busslinger, M., Shi, W., Carbone, F.R., van Lier, R.A., Kallies, A. \& van Gisbergen, K.P. Hobit and Blimp1 instruct a universal transcriptional program of tissue residency in lymphocytes. Science 352, 459-63 (2016).10.1126/science.aad2035.

48. Trapnell, C., Cacchiarelli, D., Grimsby, J., Pokharel, P., Li, S., Morse, M., Lennon, N.J., Livak, K.J., Mikkelsen, T.S. \& Rinn, J.L. The dynamics and regulators of cell fate decisions are revealed by pseudotemporal ordering of single cells. Nat Biotechnol 32, 381-386 (2014).10.1038/nbt.2859. 4122333.

49. Andreatta, M. \& Nielsen, M. Gapped sequence alignment using artificial neural networks: application to the MHC class I system. Bioinformatics 32, 511-7 (2016).10.1093/bioinformatics/btv639.

50. Nielsen, M. \& Andreatta, M. NetMHCpan-3.0; improved prediction of binding to MHC class I molecules integrating information from multiple receptor and peptide length datasets. Genome Med 8, 33 (2016).10.1186/s13073-016-0288-x. 4812631.

51. Chang, Y.F., Imam, J.S. \& Wilkinson, M.F. The nonsense-mediated decay RNA surveillance pathway. Annu Rev Biochem 76, 51-74 (2007).10.1146/annurev.biochem.76.050106.093909.

52. TCGA Research Network. Comprehensive molecular characterization of gastric adenocarcinoma. Nature 513, 202-9 (2014).10.1038/nature13480. 4170219. 
53. Le, D.T., Uram, J.N., Wang, H., Bartlett, B.R., Kemberling, H., Eyring, A.D., Skora, A.D., Luber, B.S., Azad, N.S., Laheru, D., Biedrzycki, B., Donehower, R.C., Zaheer, A., Fisher, G.A., Crocenzi, T.S., Lee, J.J., Duffy, S.M., Goldberg, R.M., de la Chapelle, A., Koshiji, M., Bhaijee, F., Huebner, T., Hruban, R.H., Wood, L.D., Cuka, N., Pardoll, D.M., Papadopoulos, N., Kinzler, K.W., Zhou, S., Cornish, T.C., Taube, J.M., Anders, R.A., Eshleman, J.R., Vogelstein, B. \& Diaz, L.A., Jr. PD-1 Blockade in Tumors with Mismatch-Repair Deficiency. N Engl J Med 372, 2509-20 (2015).10.1056/NEJMoa1500596. 4481136.

54. Yadav, M., Jhunjhunwala, S., Phung, Q.T., Lupardus, P., Tanguay, J., Bumbaca, S., Franci, C., Cheung, T.K., Fritsche, J., Weinschenk, T., Modrusan, Z., Mellman, I., Lill, J.R. \& Delamarre, L. Predicting immunogenic tumour mutations by combining mass spectrometry and exome sequencing. Nature 515, 572-6 (2014).10.1038/nature14001.

55. Carithers, L.J., Ardlie, K., Barcus, M., Branton, P.A., Britton, A., Buia, S.A., Compton, C.C., DeLuca, D.S., PeterDemchok, J., Gelfand, E.T., Guan, P., Korzeniewski, G.E., Lockhart, N.C., Rabiner, C.A., Rao, A.K., Robinson, K.L., Roche, N.V., Sawyer, S.J., Segre, A.V., Shive, C.E., Smith, A.M., Sobin, L.H., Undale, A.H., Valentino, K.M., Vaught, J., Young, T.R., Moore, H.M. \& Consortium, G.T. A Novel Approach to High-Quality Postmortem Tissue Procurement: The GTEx Project. Biopreserv Biobank 13, 311-9 (2015).10.1089/bio.2015.0032. 4675181.

56. Shukla, S.A., Rooney, M.S., Rajasagi, M., Tiao, G., Dixon, P.M., Lawrence, M.S., Stevens, J., Lane, W.J., Dellagatta, J.L., Steelman, S., Sougnez, C., Cibulskis, K., Kiezun, A., Hacohen, N., Brusic, V., Wu, C.J. \& Getz, G. Comprehensive analysis of cancer-associated somatic mutations in class I HLA genes. Nat Biotechnol 33, 1152-8 (2015).10.1038/nbt.3344. 4747795.

57. Iheagwara, U.K., Beatty, P.L., Van, P.T., Ross, T.M., Minden, J.S. \& Finn, O.J. Influenza virus infection elicits protective antibodies and T cells specific for host cell antigens also expressed as tumor-associated antigens: a new view of cancer immunosurveillance. Cancer Immunol Res 2, 263-73 (2014).10.1158/2326-6066.CIR-130125. 4006373.

58. Valkenburg, S.A., Josephs, T.M., Clemens, E.B., Grant, E.J., Nguyen, T.H., Wang, G.C., Price, D.A., Miller, A., Tong, S.Y., Thomas, P.G., Doherty, P.C., Rossjohn, J., Gras, S. \& Kedzierska, K. Molecular basis for universal HLAA*0201-restricted CD8+ T-cell immunity against influenza viruses. Proc Natl Acad Sci U S A 113, 4440-5 (2016).10.1073/pnas.1603106113. 4843436.

59. Yang, X., Chen, G., Weng, N.P. \& Mariuzza, R.A. Structural basis for clonal diversity of the human T-cell response to a dominant influenza virus epitope. J Biol Chem 292, 18618-18627 (2017).10.1074/jbc.M117.810382. 5683187.

60. Ji, Z., Song, R., Regev, A. \& Struhl, K. Many IncRNAs, 5'UTRs, and pseudogenes are translated and some are likely to express functional proteins. Elife 4, e08890 (2015).10.7554/eLife.08890. 4739776.

61. Wang, K., Singh, D., Zeng, Z., Coleman, S.J., Huang, Y., Savich, G.L., He, X., Mieczkowski, P., Grimm, S.A., Perou, C.M., MacLeod, J.N., Chiang, D.Y., Prins, J.F. \& Liu, J. MapSplice: accurate mapping of RNA-seq reads for splice junction discovery. Nucleic Acids Res 38, e178 (2010).10.1093/nar/gkq622. 2952873.

62. Jenkins, M.K. \& Moon, J.J. The role of naive T cell precursor frequency and recruitment in dictating immune response magnitude. J Immunol 188, 4135-40 (2012).10.4049/jimmunol.1102661. 3334329.

63. Li, B., Senbabaoglu, Y., Peng, W., Yang, M.L., Xu, J. \& Li, J.Z. Genomic estimates of aneuploid content in glioblastoma multiforme and improved classification. Clin Cancer Res 18, 5595-605 (2012).10.1158/10780432.CCR-12-1427. 3477792.

64. R Core Team. R: A Language and Environment for Statistical Computing. (2015).

65. Krijthe, J.H. Rtsne: T-Distributed Stochastic Neighbor Embedding using a Barnes-Hut Implementation. (2015). 